\title{
Asymptotic Theory in Model Diagnostic for General Multivariate Spatial Regression
}

\author{
Wayan Somayasa, ${ }^{1}$ Gusti N. Adhi Wibawa, ${ }^{1}$ La Hamimu, ${ }^{2}$ and La Ode Ngkoimani ${ }^{2}$ \\ ${ }^{1}$ Department of Mathematics, Haluoleo University, Kendari, Indonesia \\ ${ }^{2}$ Department of Geological Engineering, Haluoleo University, Kendari, Indonesia \\ Correspondence should be addressed to Wayan Somayasa; wayan.somayasa@uho.ac.id
}

Received 25 March 2016; Revised 13 July 2016; Accepted 28 July 2016

Academic Editor: Andrei I. Volodin

Copyright (c) 2016 Wayan Somayasa et al. This is an open access article distributed under the Creative Commons Attribution License, which permits unrestricted use, distribution, and reproduction in any medium, provided the original work is properly cited.

\begin{abstract}
We establish an asymptotic approach for checking the appropriateness of an assumed multivariate spatial regression model by considering the set-indexed partial sums process of the least squares residuals of the vector of observations. In this work, we assume that the components of the observation, whose mean is generated by a certain basis, are correlated. By this reason we need more effort in deriving the results. To get the limit process we apply the multivariate analog of the well-known Prohorov's theorem. To test the hypothesis we define tests which are given by Kolmogorov-Smirnov (KS) and Cramér-von Mises (CvM) functionals of the partial sums processes. The calibration of the probability distribution of the tests is conducted by proposing bootstrap resampling technique based on the residuals. We studied the finite sample size performance of the KS and CvM tests by simulation. The application of the proposed test procedure to real data is also discussed.
\end{abstract}

\section{Introduction}

As mentioned in the literatures of model checks for multivariate regression, the appropriateness of an assumed model is mostly verified by analyzing the least squares residual of the observations; see, for example, Zellner [1], Christensen [2], pp. 1-22, Anderson [3], pp. 187-191, and Johnson and Wichern [4], pp. 395-398. A common feature of these works is the comparison between the length of the matrix of the residuals under the null hypothesis and that of the residuals under a proposed alternative.

Instead of considering the residuals directly MacNeill [5] and MacNeill [6] proposed a method in model check for univariate polynomial regression based on the partial sums process of the residuals. These popular approaches are generalized to the spatial case by MacNeill and Jandhyala [7] for ordinary partial sums and Xie and MacNeill [8] for set-indexed partial sums process of the residuals. Bischoff and Somayasa [9] and Somayasa et al. [10] derived the limit process in the spatial case by a geometric method generalizing a univariate approach due to Bischoff [11] and Bischoff [12]. These results can be used to establish asymptotic test of Cramér-von Mises and Kolmogorov-Smirnov type for model checks and changepoint problems. Model checks for univariate regression with random design using the empirical process of the explanatory variable marked by the residuals was established in Stute [13] and Stute et al. [14]. In the papers mentioned above the limit processes were explicitly expressed as complicated functions of the univariate Brownian motion (sheet).

The purpose of the present article is to study the application of set-indexed partial sums technique to simultaneously check the goodness-of-fit of a multivariate spatial linear regression defined on high-dimensional compact rectangle. In contrast to the normal multivariate model studied in the standard literatures such as in Christensen [2], Anderson [3], and Johnson and Wichern [4] or in the references of model selection such as in Bedrick and Tsai [15] and Fujikoshi and Satoh [16], in this paper we will consider a multivariate regression model in which the components of the mean of the response vector are assumed to lie in different spaces and the underlying distribution model of the vector of random errors is unknown. 
To see the problem in more detail let $n_{1} \geq 1, \ldots, n_{d} \geq 1$ be fixed. Let a $p$-dimensional random vector $\mathbf{Z}:=\left(Z_{i}\right)_{i=1}^{p}$ be observed independently over an experimental design given by a regular lattice:

$$
\begin{aligned}
& \Xi_{n_{1} \cdots n_{d}} \\
& \quad:=\left\{\left(\frac{j_{k}}{n_{k}}\right)_{k=1}^{d} \in \mathbf{I}^{d}: 1 \leq j_{k} \leq n_{k}, k=1, \ldots, d\right\},
\end{aligned}
$$

where $\mathbf{I}^{d}:=\prod_{k=1}^{d}[0,1]$ is the $d$-dimensional unit cube. Let $\mathbf{g}:=\left(g_{i}\right)_{i=1}^{p}$ be the true but unknown $\mathbb{R}^{p}$-valued regression function on $\mathbf{I}^{d}$ which represents the mean function of the observations. Let $\mathbf{Z}_{j_{1} \cdots j_{d}}:=\left(Z_{i, j_{1} \cdots j_{d}}\right)_{i=1}^{p}$ and $\mathbf{g}:=\left(g_{i, j_{1} \cdots j_{d}}\right)_{i=1}^{p}$ be the observation and the corresponding mean in the experimental condition $\left(j_{k} / n_{k}\right)_{k=1}^{d}$. Under the null hypothesis $H_{0}$ we assume that $\mathbf{Z}_{j_{1} \cdots j_{d}}$ follows a multivariate linear model. That is, we assume a model

$$
\begin{array}{r}
H_{0}: \mathbf{Z}_{j_{1} \cdots j_{d}}=\left(\sum_{w=1}^{d_{i}} \beta_{i w} f_{i w}\left(\frac{j_{k}}{n_{k}}\right)_{k=1}^{d}\right)_{i=1}^{p}+\mathscr{E}_{j_{1} \cdots j_{d}}, \\
1 \leq j_{1} \leq n_{1}, \ldots, 1 \leq j_{d} \leq n_{d}
\end{array}
$$

where, for $i=1, \ldots, p, \beta_{i}:=\left(\beta_{i w}\right)_{w=1}^{d_{i}} \in \mathbb{R}^{d_{i}}$ is a $d_{i^{-}}$ dimensional vector of unknown parameters; $\mathbf{f}_{i}:=\left(f_{i w}\right)_{w=1}^{d_{i}}$ is a $d_{i}$-dimensional vector of known regression functions whose components are assumed to be square integrable with respect to the Lebesgue measure $\lambda_{\mathbf{I}}^{d}$ on $\mathbf{I}^{d}$, that is, $f_{i w} \epsilon$ $L_{2}\left(\lambda_{\mathrm{I}}^{d}\right)$, for all $i$ and $w . \mathscr{E}_{j_{1} \cdots j_{d}}:=\left(\varepsilon_{i, j_{1} \cdots j_{d}}\right)_{i=1}^{p}$ is the mutually independent $p$-dimensional vector of random errors defined on a common probability space $(\Omega, \mathscr{F}, \mathbb{P})$. We assume that, for all $1 \leq j_{1} \leq n_{1}, \ldots, 1 \leq j_{d} \leq n_{d}, E\left(\mathscr{E}_{j_{1} \cdots j_{d}}\right)=$ $\mathbf{0} \in \mathbb{R}^{p}$, and $\operatorname{Cov}\left(\mathscr{E}_{j_{1} \cdots j_{d}}\right)=\boldsymbol{\Sigma}=\left(\sigma_{u v}\right)_{u, v=1}^{p, p}$. Let $\mathbf{Z}_{n_{1} \cdots n_{d}}:=$ $\left(\mathbf{Z}_{j_{1} \ldots j_{d}}\right)_{j_{1}=1, \ldots, j_{d}=1}^{n_{1}, \ldots, n_{d}}$ be the $p$-dimensional pyramidal array of random observations and let $\mathscr{E}_{n_{1} \cdots n_{d}}:=\left(\mathscr{E}_{j_{1} \cdots j_{d}}\right)_{j_{1}=1, \ldots, j_{d}=1}^{n_{1}, \ldots, n_{d}}$ be the $p$-dimensional pyramidal array of random errors taking values in the Euclidean space $\prod_{i=1}^{p} \mathbb{R}^{n_{1} \times \cdots \times n_{d}}$. Then under $H_{0}$ the observations can be represented by

$$
\mathbf{Z}_{n_{1} \cdots n_{d}}=\left(\sum_{w=1}^{d_{i}} \beta_{i w} f_{i w}\left(\Xi_{n_{1} \cdots n_{d}}\right)\right)_{i=1}^{p}+\mathscr{E}_{n_{1} \cdots n_{d}},
$$

where $f_{i w}\left(\Xi_{n_{1} \cdots n_{d}}\right):=\left(f_{i w}\left(\left(j_{k} / n_{k}\right)_{k=1}^{d}\right)\right)_{j_{1}=1, \ldots, j_{d}=1}^{n_{1}, n_{d}}$. Under the alternative $H_{1}$ a multivariate nonparametric regression model

$$
\mathbf{Z}_{n_{1} \cdots n_{d}}=\mathbf{g}\left(\Xi_{n_{1} \cdots n_{d}}\right)+\mathscr{E}_{n_{1} \cdots n_{d}}
$$

is assumed, where $\mathbf{g}\left(\Xi_{n_{1} \cdots n_{d}}\right):=\left(\mathbf{g}\left(\left(j_{k} / n_{k}\right)_{k=1}^{d}\right)\right)_{j_{1}=1, \ldots, j_{d}=1}^{n_{1}, \ldots, n_{d}} \in$ $\prod_{i=1}^{p} \mathbb{R}^{n_{1} \times \cdots \times n_{d}}$. By applying the similar argument as in Christensen [2] and Johnson and Wichern [4], the $p$-dimensional array of the least squares residuals of the observations is given by the following component-wise projection:

$$
\begin{aligned}
\mathbf{R}_{n_{1} \cdots n_{d}} & :=\left(\mathbf{r}_{j_{1} \cdots j_{d}}\right)_{j_{1}, \ldots, j_{d}=1}^{n_{1}, \ldots, n_{d}} \\
& :=\mathbf{Z}_{n_{1} \cdots n_{d}}-\operatorname{pr}_{\prod_{i=1}^{p} \mathbf{w}_{i, n_{1} \cdots n_{d}}} \mathbf{Z}_{n_{1} \cdots n_{d}},
\end{aligned}
$$

with $\mathbf{r}_{j_{1} \cdots j_{d}}:=\left(r_{i, j_{1} \cdots j_{d}}\right)_{i=1}^{p}$, for $1 \leq j_{k} \leq n_{k}$, and $k=$ $1, \ldots, d$. Thereby, for $i=1, \ldots, p$, we define $\mathbf{W}_{i, n_{1} \cdots n_{d}}:=$ $\left[f_{i 1}\left(\Xi_{n_{1} \cdots n_{d}}\right), \ldots, f_{i d_{i}}\left(\Xi_{n_{1} \cdots n_{d}}\right)\right]$ as the subspace of $\mathbb{R}^{n_{1} \times \cdots \times n_{d}}$ spanned by the arrays $\left\{f_{i 1}\left(\Xi_{n_{1} \cdots n_{d}}\right), \ldots, f_{i d_{i}}\left(\Xi_{n_{1} \cdots n_{d}}\right)\right\}$. It is worth mentioning that the Euclidean space $\mathbb{R}^{n_{1} \times \cdots \times n_{d}}$ is furnished with the inner product denoted by $\langle\cdot, \cdot\rangle_{\mathbb{R}^{n_{1} \times \cdots \times n_{d}}}$ and defined by

$$
\left\langle\mathbf{A}_{n_{1} \cdots n_{d}}, \mathbf{B}_{n_{1} \cdots n_{d}}\right\rangle_{\mathbb{R}^{n_{1} \times \cdots \times n_{d}}}:=\sum_{j_{1}=1}^{n_{1}} \cdots \sum_{j_{d}=1}^{n_{d}} a_{j_{1} \cdots j_{d}} b_{j_{1} \cdots j_{d}},
$$

for every $\mathbf{A}_{n_{1} \cdots n_{d}}:=\left(a_{j_{1} \cdots j_{d}}\right)_{j_{1}=1, \ldots, j_{d}=1}^{n_{1}, \ldots, n_{d}}$, and $\mathbf{B}_{n_{1} \cdots n_{d}}:=$ $\left(b_{j_{1} \cdots j_{d}}\right)_{j_{1}=1, \ldots, j_{d}=1}^{n_{1}, \ldots, n_{d}} \in \mathbb{R}^{n_{1} \times \cdots \times n_{d}}$.

Next we define the set-indexed partial sums operator. Let $\mathscr{A}$ be the family of convex subset of $\mathrm{I}^{d}$, and let $\eta_{\lambda_{\mathrm{I}}^{d}}$ be the Lebesgue pseudometric on $\mathscr{A}$ defined by $\eta_{\lambda_{\mathrm{I}}^{d}}\left(A_{1}, A_{2}\right):=$ $\lambda_{\mathrm{I}}^{d}\left(A_{1} \Delta A_{2}\right)$, for $A_{1}, A_{2} \in \mathscr{A}$. Let $\mathscr{C}(\mathscr{A})$ be the set of continuous functions on $\mathscr{A}$ under $\eta_{\lambda_{\mathrm{I}}^{d}}$. We embed the array of the residual $\mathbf{R}_{n_{1} \cdots n_{d}}$ into a $p$-dimensional stochastic process indexed by $\mathscr{A}$ by using the component-wise set-indexed partial sums operator

$$
\mathbf{V}_{n_{1} \cdots n_{d}}: \prod_{i=1}^{p} \mathbb{R}^{n_{1} \times \cdots \times n_{d}} \longmapsto \mathscr{C}^{p}(\mathscr{A}):=\prod_{i=1}^{p} \mathscr{C}(\mathscr{A}),
$$

such that, for any $B \in \mathscr{A}$,

$$
\begin{aligned}
& \mathbf{V}_{n_{1} \cdots n_{d}}\left(\mathbf{R}_{n_{1} \cdots n_{d}}\right)(B) \\
& \quad:=\sum_{j_{1}=1}^{n_{1}} \cdots \sum_{j_{d}=1}^{n_{d}} \sqrt{n_{1} \cdots n_{d}} \lambda_{\mathbf{I}}^{d}\left(B \cap C_{j_{1} \cdots j_{d}}\right) \mathbf{r}_{j_{1} \cdots j_{d}},
\end{aligned}
$$

where, for $1 \leq j_{1} \leq n_{1}, \ldots, 1 \leq j_{d} \leq n_{d}, C_{j_{1} \ldots j_{d}}:=\prod_{k=1}^{d}\left(\left(j_{k}-\right.\right.$ $\left.1) / n_{k}, j_{k} / n_{k}\right]$. Let us call this process the $p$-dimensional setindexed least squares residual partial sums process. The space $\mathscr{C}^{P}(\mathscr{A})$ is furnished with the uniform topology induced by the metric $\varphi$ defined by

$$
\varphi(\mathbf{u}, \mathbf{w}):=\sum_{i=1}^{p}\left\|u_{i}-w_{i}\right\|_{\mathscr{A}}=\sum_{i=1}^{p} \sup _{A \in \mathscr{A}}\left|u_{i}(A)-w_{i}(A)\right|,
$$

for $\mathbf{u}:=\left(u_{i}\right)_{i=1}^{p}$ and $\mathbf{w}:=\left(w_{i}\right)_{i=1}^{p} \in \mathscr{C}^{p}(\mathscr{A})$.

We notice that, in the works of Bischoff and Somayasa [9], Bischoff and Gegg [17], and Somayasa and Adhi Wibawa [18], the limit process of the partial sums process of the least squares residuals has been investigated by applying the existing geometric method of Bischoff $[11,12]$. However, the method becomes not applicable anymore in deriving the limit process of $\mathbf{V}_{n_{1} \cdots n_{d}}\left(\mathbf{R}_{n_{1} \cdots n_{d}}\right)$ as the dimension of $\mathbf{W}_{i, n_{1} \cdots n_{d}}$ varies. Therefore, in this work, we attempt to adopt the vectorial analog of Prohorov's theorem; see, for example, Theorem 6.1 in Billingsley [19] for obtaining the limit process. For our result we need to extend the ordinary partial sums formula to $p$-dimensional case defined on $\mathbf{I}^{d}$ as follows. Let $K$ := $\{1,2, \ldots, d\}$ and $C_{k}^{K}$ be the set of all $k$-combinations of the set $K$, with $k=1, \ldots, d$. For a chosen value of $k$, we denote 
the $i$ th element of $C_{k}^{K}$ by a $k$-tuple $\left(i\left(k_{1}\right), i\left(k_{2}\right), \ldots, i\left(k_{k}\right)\right)$, for $1 \leq i \leq\left|C_{k}^{K}\right|$, where $\left|C_{k}^{K}\right|$ is the number of elements of $C_{k}^{K}$ which is clearly given by

$$
\left|C_{k}^{K}\right|=\frac{d(d-1)(d-2) \cdots(d-k+1)}{k(k-1)(k-2) \cdots 1} .
$$

For example, let $K=\{1,2,3\}$. Then, for $k=1$, we denote the elements of $C_{1}^{K}$ as $1\left(k_{1}\right):=1,2\left(k_{1}\right):=2$, and $3\left(k_{1}\right):=3$. In a similar way, we denote the elements of $C_{2}^{K}$ which consists of $\{(1,2),(1,3),(2,3)\}$, respectively, by $\left(1\left(k_{1}\right), 1\left(k_{2}\right)\right):=(1,2)$, $\left(2\left(k_{1}\right), 2\left(k_{2}\right)\right):=(1,3)$, and $\left(3\left(k_{1}\right), 3\left(k_{2}\right)\right):=(2,3)$. Finally the element of $C_{3}^{K}=\{(1,2,3)\}$ is sufficiently written by $\left(1\left(k_{1}\right), 1\left(k_{2}\right), 1\left(k_{3}\right)\right)$. Hence the $p$-dimensional ordinary partial sums operator transforms any $p$-dimensional array $\mathbf{A}_{n_{1} \cdots n_{d}}=\left(\mathbf{a}_{j_{1} \cdots j_{d}}\right)_{j_{1}=1, \ldots, j_{d}=1}^{n_{1}, \ldots, n_{d}} \in \prod_{i=1}^{p} \mathbb{R}^{n_{1} \times \cdots \times n_{d}}$ to a continuous function on $\mathbf{I}^{d}$ defined by

$$
\begin{aligned}
& \mathbf{T}_{n_{1} \cdots n_{d}}\left(\mathbf{A}_{n_{1} \cdots n_{d}}\right)(\mathbf{t}):=\frac{1}{\sqrt{n_{1} \cdots n_{d}}} \sum_{j_{1}=1 \cdots j_{d}=1}^{\left[n_{1} t_{1}\right] \cdots\left[n_{d} t_{d}\right]} \mathbf{a}_{j_{1} \cdots j_{d}} \\
& +\sum_{k=1}^{d} \sum_{i=1}^{\left|C_{2}^{K}\right|}\left(\prod_{u=1}^{k}\left(t_{i\left(k_{u}\right)}-\frac{\left[n_{i\left(k_{u}\right)} t_{i\left(k_{u}\right)}\right]}{\left.n_{i\left(k_{u}\right)}\right)}\right)\right) \\
& \cdot \frac{\sqrt{n_{1} \cdots n_{d}}}{\left(n_{1} \cdots n_{d}\right) /\left(n_{i\left(k_{1}\right)} \cdots n_{i\left(k_{d}\right)}\right)} \\
& \cdot \sum_{\left\{j_{1}=1, \ldots, j_{d}=1\right\} /\left\langle j_{\left.i\left(k_{1}\right), \ldots, j_{i\left(k_{d}\right)}\right\}} \mathbf{a}_{j_{1} \cdots j_{d} \mid j_{i\left(k_{1}\right)}=\left[n_{i\left(k_{1}\right)} t_{i\left(k_{1}\right)}\right]+1, \ldots, j_{i\left(k_{d}\right)}=\left[n_{i\left(k_{d}\right)} t_{i\left(k_{d}\right)}\right]+1},\right.}
\end{aligned}
$$

for every $\mathbf{t}:=\left(t_{1}, \ldots, t_{d}\right)^{\top} \in \mathbf{I}^{d}$, where for positive integers $b_{u}, b_{u+1}, \ldots, b_{u+m} \in \mathscr{Z}_{+}$we define a notation

$$
\begin{aligned}
\mathbf{a}_{j_{1} \ldots j_{d}} \mid j_{u} & =b_{u}, \ldots, j_{u+m}=b_{u+m} \\
& :=\mathbf{a}_{j_{1}, \ldots, j_{u-1}, b_{u}, \ldots, b_{u+m}, j_{u+m+1}, \ldots, j_{d}} .
\end{aligned}
$$

It is clear that the partial sums process of the residuals obtained using (11) is a special case of (8) since for every $\mathbf{t}:=\left(t_{1}, \ldots, t_{d}\right)^{\top} \in \mathbf{I}^{d}$ it holds

$$
\mathbf{T}_{n_{1} \cdots n_{d}}\left(\mathbf{A}_{n_{1} \cdots n_{d}}\right)(\mathbf{t})=\mathbf{V}_{n_{1} \cdots n_{d}}\left(\mathbf{A}_{n_{1} \cdots n_{d}}\right)\left(\prod_{k=1}^{d}\left[0, t_{k}\right]\right) .
$$

It is worth noting that the extension of the study from univariate to multivariate model and also the expansion of the dimension of the lattice points are strongly motivated by the prediction problem in mining industry and geosciences. As for an example recently Tahir [20] presented data provided by PT Antam Tbk (a mining industry in Southeast Sulawesi). The data consist of a joint measurement of the percentage of several chemical elements and substances such as $\mathrm{Ni}, \mathrm{Co}$, $\mathrm{Fe}, \mathrm{MgO}, \mathrm{SiO}_{2}$, and $\mathrm{CaO}$ which are recorded in every point of a three-dimensional lattice defined over the exploration region of the company. Hence, by the inherent existence of the correlation among the variables, the statistical analysis for the involved variables must be conducted simultaneously.
There have been many methods proposed in the literatures for testing $H_{0}$. Most of them have been derived for the case of $\mathbf{W}_{1}=\cdots=\mathbf{W}_{p}$ under normally distributed random error. Generalized likelihood ratio test which has been leading to Wilk's lambda statistic or variant of it can be found in Zellner [1], Christensen [2], pp. 1-22, Anderson [3], pp. 187-191, and Johnson and Wichern [4], pp. 395-398. Mardia and Goodall [21] derived the maximum likelihood estimation procedure for the parameters of the general normal spatial multivariate model with stationary observations. This approach can be straightforwardly extended for obtaining the associated likelihood ratio test in model check for the model. Unfortunately, in the practice especially when dealing with mining data, normal distribution is sometimes found to be not suitable for describing the distribution model of the observations, so that the test procedures mentioned above are consequently no longer applicable. Asymptotic method established in Arnold [22] for multivariate regression with $\mathbf{W}_{1}=\cdots=\mathbf{W}_{p}$ can be generalized in such a way that it is valid for the general model. As a topic in statistics it must be well known. However, we cannot find literatures where the topic has been studied.

The rest of the paper is organized as follows. In Section 2 we show that when $H_{0}$ is true $\Sigma^{-1 / 2} \mathbf{V}_{n_{1} \cdots n_{d}}\left(\mathbf{R}_{n_{1} \cdots n_{d}}\right)$ converges weakly to a projection of the $p$-dimensional set-indexed Brownian sheet. The limit process is shown to be useful for testing $\mathrm{H}_{0}$ asymptotically based on the Kolmogorov-Smirnov (KS-test) and Cramér-von Mises (CvM-test) functionals of the set-indexed $p$-dimensional least squares residual partial sum processes, defined, respectively, by

$$
\begin{aligned}
& \mathrm{KS}_{n_{1} \cdots n_{d}, \mathscr{A}}:=\sup _{A \in \mathscr{A}}\left\|\Sigma^{-1 / 2} \mathbf{V}_{n_{1} \cdots n_{d}}\left(\mathbf{R}_{n_{1} \cdots n_{d}}\right)(A)\right\|_{\mathbb{R}^{p}} \\
& \mathrm{CvM}_{n_{1} \cdots n_{d}, \mathscr{A}} \\
& \quad:=\frac{1}{n_{1} \cdots n_{d}} \sum_{A \in \mathscr{A}}\left\|\Sigma^{-1 / 2} \mathbf{V}_{n_{1} \cdots n_{d}}\left(\mathbf{R}_{n_{1} \cdots n_{d}}\right)(A)\right\|_{\mathbb{R}^{p}}^{2} .
\end{aligned}
$$

For both tests the rejection of $H_{0}$ is for large value of the KS and CvM statistics, respectively. Under localized alternative the above sequence of random processes converges weakly to the above limit process with an additional deterministic trend (see Section 3). In Section 4, we define a consistent estimator for $\Sigma$. In Section 5 we investigate a residual based bootstrap method for the calibration of the tests. Monte Carlo simulation for the purpose of studying the finite sample behavior of the KS and CvM tests is reported in Section 6. Application of the test procedure in real data is presented in Section 7. The paper is closed in Section 8 with conclusion and some remarks for future research. Auxiliary results needed for deriving the limit process are presented in the appendix. We note that all convergence results derived throughout this paper which hold for $n_{1}, \ldots, n_{d}$ simultaneously go to infinity, that is, for $n_{k} \rightarrow \infty$, for all $k=1, \ldots, d$; otherwise they will be stated in some way. The notion of convergence in distribution and convergence in probability will be conventionally denoted by $\stackrel{\mathscr{D}}{\longrightarrow}$ and $\stackrel{\mathscr{P}}{\longrightarrow}$, respectively. 


\section{The Limit of $\mathbf{V}_{n_{1} \cdots n_{d}}\left(\mathbf{R}_{n_{1} \cdots n_{d}}\right)$ under $H_{0}$}

Let $B:=\{B(A): A \in \mathscr{A}\}$ be the one-dimensional set-indexed Brownian sheet having sample path in $\mathscr{C}(\mathscr{A})$. We refer the reader to Pyke [23], Bass and Pyke [24], and Alexander and Pyke [25] for the definition and the existence of $B$. Let us consider a subspace of $\mathscr{C}(\mathscr{A})$ which is closely related to $B$, defined by

$$
\begin{aligned}
\mathscr{H}_{B} & :=\left\{h_{v}: \mathscr{A} \longrightarrow \mathbb{R}, \exists v \in L_{2}\left(\lambda_{\mathrm{I}}^{d}\right), \text { s.t. } h_{v}(A)\right. \\
& \left.:=\int_{A} v(\mathbf{t}) \lambda^{d}(\mathbf{t})\right\} .
\end{aligned}
$$

Under the inner product and the norm defined by

$$
\begin{aligned}
\left\langle h_{v}, h_{w}\right\rangle_{\mathscr{H}_{B}} & :=\int_{\mathbf{I}^{d}} v(\mathbf{t}) w(\mathbf{t}) \lambda_{\mathbf{I}}^{d}(\mathbf{t}), \\
\left\|h_{v}\right\|^{2} & :=\int_{\mathbf{I}^{d}} v^{2}(\mathbf{t}) \lambda_{\mathbf{I}}^{d}(\mathbf{t}),
\end{aligned}
$$

it is clear that $\mathscr{H}_{B}$ and $L_{2}\left(\lambda_{\mathrm{I}}^{d}\right)$ are isometric. For our result we need to define subspaces $\mathbf{W}_{i}$ and $\mathbf{W}_{i \mathscr{H}_{B}}$ associated with the regression functions $f_{i 1}, \ldots, f_{i d_{i}}$, where $\mathbf{W}_{i}:=\left[f_{i 1}, \ldots, f_{i d_{i}}\right] \subset$ $L_{2}\left(\lambda_{\mathrm{I}}^{d}\right)$ and $\mathbf{W}_{i \mathscr{H}_{B}}:=\left[h_{f_{i 1}}, \ldots, h_{f_{i d_{i}}}\right] \subset \mathscr{H}_{B}$, for $i=1, \ldots, p$.

Now we are ready to state the limit process of the sequence of $p$-dimensional set-indexed residual partial sums processes for the model specified under $H_{0}$.

Theorem 1. For $i=1, \ldots, p$, let $\left\{f_{i 1}, \ldots, f_{i d_{i}}\right\}$ be an orthonormal basis $(O N B)$ of $\mathbf{W}_{i}$. We assume that $\mathbf{W}_{i} \subseteq \mathbf{W}_{i+1}$, for $i=1, \ldots, p-1$. Let $\mathbf{B}_{p}:=\left\{\left(B_{i}(A)\right)_{i=1}^{p}: A \in \mathscr{A}\right\}$ be the $p$-dimensional set-indexed Brownian sheet with the covariance function $\operatorname{Cov}\left(\mathbf{B}_{p}(A), \mathbf{B}_{p}\left(A^{\prime}\right)\right):=\lambda_{\mathbf{I}}^{d}\left(A \cap A^{\prime}\right) \mathbf{I}_{p}$, for $A, A^{\prime} \in \mathscr{A}$, where $\mathbf{I}_{p}$ is the $p \times p$ identity matrix. Suppose $\left\{f_{i 1}, \ldots, f_{i d_{i}}\right\}$ are in $\mathscr{C}\left(\mathbf{I}^{d}\right) \cap \mathrm{BV}_{H}\left(\mathbf{I}^{d}\right)$, where $\mathscr{C}\left(\mathbf{I}^{d}\right)$ is the space of continuous functions on $\mathbf{I}^{d}$ (see Definition A.4 for the definition of $\mathrm{BV}_{H}\left(\mathbf{I}^{d}\right)$ ). Then under $H_{0}$ it holds that

$$
\Sigma^{-1 / 2} \mathbf{V}_{n_{1} \cdots n_{d}}\left(\mathbf{R}_{n_{1} \cdots n_{d}}\right) \stackrel{\mathscr{D}}{\longrightarrow} \mathbf{B}_{p, \mathbf{f}}^{H_{0}}:=\mathbf{B}_{p}-p r_{\prod_{i=1}^{p} \mathbf{w}_{i \mathscr{C}_{B}}}^{*} \mathbf{B}_{p}
$$

where

$$
\operatorname{pr}_{\prod_{i=1}^{p} \mathbf{w}_{i \mathscr{H}_{B}}}^{*} \mathbf{B}_{p}:=\left(\operatorname{pr}_{\mathbf{W}_{i \mathscr{H}_{B}}}^{*} B_{i}\right)_{i=1}^{p}
$$

Thereby $\operatorname{pr}_{\mathbf{W}_{i \mathscr{H}_{B}}}^{*}$ is a projector such that, for every $u \in \mathscr{C}(\mathscr{A})$ and $A \in \mathscr{A}$,

$$
\begin{aligned}
\left(\operatorname{pr}_{\mathbf{W}_{i \mathscr{H}_{B}}}^{*} u\right)(A):=\sum_{j=1}^{d_{i}}\left\langle h_{f_{i j}}, u\right\rangle h_{f_{i j}}(A) \\
\text { where }\left\langle h_{f_{i j}}, u\right\rangle:=\int_{\mathrm{I}^{d}}^{(R)} f_{i j}(\mathbf{t}) d u(\mathbf{t}) .
\end{aligned}
$$

For $\mathbf{t}:=\left(t_{k}\right)_{k=1}^{d} \in \mathbf{I}^{d}$, we set $u(\mathbf{t})$ for $u\left(\prod_{k=1}^{d}\left[0, t_{k}\right]\right)$, and $\int^{(R)}$ stands for the integral in the sense of Riemann-Stieltjes.
Moreover, the limit process $\mathbf{B}_{p, \mathbf{f}}^{H_{0}}$ is a centered Gaussian process with the covariance function given by

$$
\begin{aligned}
& K(A, C):=\operatorname{diag}(\lambda(A \cap C) \\
& \quad-\sum_{j=1}^{d_{1}} h_{f_{1 j}}(A) h_{f_{1 j}}(C), \ldots, \lambda(A \cap C) \\
& \left.-\sum_{j=1}^{d_{p}} h_{f_{p j}}(A) h_{f_{p j}}(C)\right) .
\end{aligned}
$$

Proof. By applying the linear property of $\mathbf{V}_{n_{1} \cdots n_{d}}$ and Lemma C.2, we have under $H_{0}$,

$$
\begin{aligned}
\mathbf{V}_{n_{1} \cdots n_{d}}\left(\mathbf{R}_{n_{1} \cdots n_{d}}\right)= & \mathbf{V}_{n_{1} \cdots n_{d}}\left(\mathscr{E}_{n_{1} \cdots n_{d}}\right) \\
& -\operatorname{pr}_{\prod_{i=1}^{p} \mathbf{W}_{i, n_{1} \cdots n_{d} \mathscr{H}_{B}}} \mathbf{V}_{n_{1} \cdots n_{d}}\left(\mathscr{E}_{n_{1} \cdots n_{d}}\right) .
\end{aligned}
$$

It can be shown by extending the uniform central limit theorem studied in Pyke [23], Bass and Pyke [24], and Alexander and Pyke [25] to its vectorial analog that the term $\Sigma^{-1 / 2} \mathbf{V}_{n_{1} \cdots n_{d}}\left(\mathscr{E}_{n_{1} \cdots n_{d}}\right)$ on the right-hand side of (21) converges weakly to $\mathbf{B}_{p}$. Therefore we only need to show that the second term satisfies the weak convergence:

$$
\begin{aligned}
\mathscr{Z}_{n_{1} \cdots n_{d}} & :=\boldsymbol{\Sigma}^{-1 / 2} \operatorname{pr}_{\prod_{i=1}^{p} \mathbf{w}_{i, n_{1} \cdots n_{d} \mathscr{\mathscr { H } _ { B }}}} \mathbf{V}_{n_{1} \cdots n_{d}}\left(\mathscr{E}_{n_{1} \cdots n_{d}}\right) \stackrel{\mathscr{D}}{\longrightarrow} \\
U & :=\operatorname{pr}_{\prod_{i=1}^{*} \mathbf{w}_{i \mathscr{H}_{B}}} \mathbf{B}_{p},
\end{aligned}
$$

where $U$ is a $p$-dimensional centered Gaussian process with the covariance matrix given by

$$
\begin{gathered}
K_{U}\left(A_{1}, A_{2}\right)=\operatorname{diag}\left(\sum_{j=1}^{d_{1}} h_{f_{1 j}}\left(A_{1}\right) h_{f_{1 j}}\left(A_{2}\right), \ldots,\right. \\
\left.\sum_{j=1}^{d_{p}} h_{f_{p j}}\left(A_{1}\right) h_{f_{p j}}\left(A_{2}\right)\right), \quad \text { for } A_{1}, A_{2} \in \mathscr{A} .
\end{gathered}
$$

By Prohorov's theorem it is sufficient to show that, for any finite collection of convex sets $A_{1}, \ldots, A_{r}$ in $\mathscr{A}$ and real numbers $c_{1}, \ldots, c_{r}$, with $r \geq 1$, it holds that

$$
\sum_{k=1}^{r} c_{k} \mathscr{Z}_{n_{1} \cdots n_{d}}\left(A_{k}\right) \stackrel{\mathscr{D}}{\longrightarrow} \sum_{k=1}^{r} c_{k} U\left(A_{k}\right),
$$

where the left-hand side has the covariance which can be expressed as

$$
\begin{aligned}
\operatorname{Cov} & \left(\sum_{k=1}^{r} c_{k} \mathscr{Z}_{n_{1} \cdots n_{d}}\left(A_{k}\right)\right) \\
= & \sum_{k=1}^{r} \sum_{\ell=1}^{r} c_{k} c_{\ell} \Sigma^{-1 / 2}\left(E\left(B_{i}\left(A_{k}\right) B_{j}\left(A_{\ell}\right)\right)\right)_{i, j=1}^{p} \Sigma^{-1 / 2},
\end{aligned}
$$


where $B_{i}\left(A_{k}\right):=\operatorname{pr}_{\mathbf{W}_{i, n_{1} \cdots n_{d} \mathscr{B} \mathcal{E}_{B}}} \mathbf{V}_{n_{1} \cdots n_{d}}\left(\varepsilon_{i, n_{1} \cdots n_{d}}\right)\left(A_{k}\right)$, for $i=$ $1, \ldots, p$ and $k=1, \ldots, r$. Let $k$ and $\ell$ be fixed. Then by

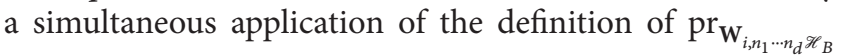
(see Lemma C.3), (11), the definition of the Riemann-Stieljes integral (cf. Stroock [26], pp. 7-17), and the independence of the array $\left\{\varepsilon_{i, j_{1} \cdots j_{d}}: 1 \leq j_{k} \leq n_{k}\right\}$, we further get

$$
\begin{gathered}
E\left(B_{i}\left(A_{k}\right) B_{j}\left(A_{\ell}\right)\right)=\sum_{w=1}^{d_{i}} \sum_{w^{\prime}=1}^{d_{j}} \int_{A_{k}} \widetilde{s}_{i w}^{\left(n_{1} \cdots n_{d}\right)}(\mathbf{t}) \lambda_{\mathbf{I}}^{d}(d \mathbf{t}) \\
\cdot \int_{A_{\ell}} \widetilde{s}_{j w^{\prime}}^{\left(n_{1} \cdots n_{d}\right)}(\mathbf{t}) \lambda_{\mathbf{I}}^{d}(d \mathbf{t}) E\left(\int_{I^{d}}^{(R)} \widetilde{s}_{i w}^{\left(n_{1} \cdots n_{d}\right)}(\mathbf{t}) d \mathbf{V}_{n_{1} \cdots n_{d}}\right. \\
\left.\cdot\left(\varepsilon_{i, n_{1} \cdots n_{d}}\right)(\mathbf{t}) \int_{\mathbf{I}^{d}}^{(R)} \widetilde{s}_{j w^{\prime}}^{\left(n_{1} \cdots n_{d}\right)}(\mathbf{t}) d \mathbf{V}_{n_{1} \cdots n_{d}}\left(\varepsilon_{j, n_{1} \cdots n_{d}}\right)(\mathbf{t})\right) \\
=\sum_{w=1}^{d_{i}} \sum_{w^{\prime}=1}^{d_{j}} \int_{A_{k}} \widetilde{s}_{i w}^{\left(n_{1} \cdots n_{d}\right)}(\mathbf{t}) \lambda_{\mathbf{I}}^{d}(d \mathbf{t}) \int_{A_{\ell}} \widetilde{s}_{j w^{\prime}}^{\left(n_{1} \cdots n_{d}\right)}(\mathbf{t}) \\
\cdot \lambda_{\mathbf{I}}^{d}(d \mathbf{t}) \frac{\sigma_{i j}}{n_{1} \cdots n_{d}} \sum_{j_{1}=1, \ldots, j_{d}=1}^{n_{1}, \cdots, n_{d}} \widetilde{s}_{i w}^{\left(n_{1} \cdots n_{d}\right)}\left(\frac{j_{1}}{n_{1}}, \ldots, \frac{j_{d}}{n_{d}}\right) \\
\cdot \widetilde{s}_{j w^{\prime}}^{\left(n_{1} \cdots n_{d}\right)}\left(\frac{j_{1}}{n_{1}}, \ldots, \frac{j_{d}}{n_{d}}\right) .
\end{gathered}
$$

By recalling Lemma C.3 the last expression clearly converges to

$$
\begin{aligned}
& \sigma_{i j} \sum_{w=1}^{d_{i}} \sum_{w^{\prime}=1}^{d_{j}}\left\langle f_{i w}, f_{j w^{\prime}}\right\rangle_{L_{2}\left(\lambda_{\mathrm{I}}^{d}\right)} h_{f_{i w}}\left(A_{k}\right) h_{f_{j w^{\prime}}}\left(A_{\ell}\right) \\
& \quad=\sigma_{i j} \sum_{w=1}^{d_{i}} h_{f_{i w}}\left(A_{k}\right) h_{f_{j w}}\left(A_{\ell}\right) .
\end{aligned}
$$

Hence the matrix $\left(E\left(B_{i}\left(A_{k}\right) B_{j}\left(A_{\ell}\right)\right)\right)_{i, j=1}^{p}$ converges element wise to the symmetric matrix which can be represented as $\Sigma \odot$ $\mathbf{D}$, for a matrix $\mathbf{D}$ defined by

D

$$
:=\left(\begin{array}{cccc}
\kappa_{11}\left(A_{k}, A_{\ell}\right) & \kappa_{12}\left(A_{k}, A_{\ell}\right) & \cdots & \kappa_{1 p}\left(A_{k}, A_{\ell}\right) \\
\kappa_{21}\left(A_{k}, A_{\ell}\right) & \kappa_{22}\left(A_{k}, A_{\ell}\right) & \cdots & \kappa_{2 p}\left(A_{k}, A_{\ell}\right) \\
\vdots & \vdots & \vdots & \vdots \\
\kappa_{p 1}\left(A_{k}, A_{\ell}\right) & \kappa_{p 2}\left(A_{k}, A_{\ell}\right) & \cdots & \kappa_{p p}\left(A_{k}, A_{\ell}\right)
\end{array}\right)
$$

with $\kappa_{i j}\left(A_{k}, A_{\ell}\right):=\sum_{w=1}^{d_{\min \{i, j\}}} h_{f_{i w}}\left(A_{k}\right) h_{f_{j w}}\left(A_{\ell}\right)$, for $i, j=$ $1, \ldots, p$. Thereby $\odot$ denotes the Hadarmard product defined, for example, in Magnus and Neudecker [27], pp. 53-54. Since $\boldsymbol{\Sigma}^{-1 / 2}(\boldsymbol{\Sigma} \odot \mathbf{D}) \boldsymbol{\Sigma}^{-1 / 2}=\mathbf{D} \odot \mathbf{I}_{p}$, we successfully have shown that $\operatorname{Cov}\left(\sum_{k=1}^{r} c_{k} \mathscr{X}_{n_{1} \cdots n_{d}}\left(A_{k}\right)\right)$ converges to the following general linear combination:

$$
\begin{aligned}
& \sum_{k=1}^{r} \sum_{\ell=1}^{r} c_{k} c_{\ell} \\
& \quad \cdot \operatorname{diag}\left(\sum_{w=1}^{d_{1}} h_{f_{1 w}}\left(A_{k}\right) h_{f_{1 w}}\left(A_{\ell}\right), \ldots, \sum_{w=1}^{d_{p}} h_{f_{p w}}\left(A_{k}\right) h_{f_{p w}}\left(A_{\ell}\right)\right),
\end{aligned}
$$

which is actually the covariance of $\sum_{k=1}^{r} c_{k} U\left(A_{k}\right)$. Next we observe that, by applying the definition of $\mathbf{V}_{n_{1} \cdots n_{d}}$ and the definition of the Riemann-Stieltjes integral, we can also write $\sum_{k=1}^{r} c_{k} \mathscr{E}_{n_{1} \cdots n_{d}}\left(A_{k}\right)$ as follows:

$$
\begin{aligned}
& \sum_{k=1}^{r} \mathcal{c}_{k} \mathscr{X}_{n_{1} \cdots n_{d}}\left(A_{k}\right) \\
& =\sum_{j_{1}=1}^{n_{1}} \cdots \sum_{j_{d}=1}^{n_{d}} \Sigma^{-1 / 2}\left(\sum_{k=1}^{r} \Lambda_{i}\left(\frac{j_{1}}{n_{1}}, \ldots, \frac{j_{d}}{n_{d}} ; A_{k}\right)\right)_{i=1}^{p},
\end{aligned}
$$

where

$$
\begin{aligned}
& \Lambda_{i}\left(\frac{j_{1}}{n_{1}}, \ldots, \frac{j_{d}}{n_{d}} ; A_{k}\right):=\sum_{w=1}^{d_{i}} \frac{c_{k}}{\sqrt{n_{1} \cdots n_{d}}} \\
& \cdot \widetilde{s}_{i w}^{\left(n_{1} \cdots n_{d}\right)}\left(\frac{j_{1}}{n_{1}}, \ldots, \frac{j_{d}}{n_{d}}\right) \varepsilon_{i, j_{1} \cdots j_{d}} h_{\widetilde{s}_{i w}^{\left(n_{1} \cdots n_{d}\right)}}\left(A_{k}\right) .
\end{aligned}
$$

Let $\Gamma_{f}:=\max _{1 \leq w \leq d_{i} ; 1 \leq i \leq p}\left\|f_{i w}\right\|_{\infty}, M:=\max _{1 \leq k \leq r}\left|\mathcal{c}_{k}\right|$, and $\left\|\Sigma^{-1 / 2}\right\|$ be the Euclidean norm of $\Sigma^{-1 / 2}$. Then by considering the stochastic independence of the array of the $p$-vector of the random errors, it holds that

$$
\begin{aligned}
& 0 \leq \mathbb{L}_{\mathscr{E}_{n_{1} \cdots n_{d}}}(\epsilon):=\sum_{j_{1}=1}^{n_{1}} \ldots \sum_{j_{d}=1}^{n_{d}} E \\
& \cdot\left(\left\|\left(\sum_{k=1}^{r} \Lambda_{i}\left(\frac{j_{1}}{n_{1}}, \ldots, \frac{j_{d}}{n_{d}} ; A_{k}\right)\right)_{i=1}^{p}\right\|_{\mathbb{R}^{p}}^{2}\right. \\
&\left.\cdot \mathbf{1}\left\{\left\|\left(\sum_{k=1}^{r} \Lambda_{i}\left(\frac{j_{1}}{n_{1}}, \ldots, \frac{j_{d}}{n_{d}} ; A_{k}\right)\right)_{i=1}^{p}\right\|_{\mathbb{R}^{p}}^{2} \geq \epsilon\right\}\right) \\
& \leq(r M)^{2}\left(d_{p} \Gamma_{f}^{2}\right)^{2}\left\|\Sigma-\frac{1}{2}\right\|^{2} E\left(\left\|\mathscr{E}_{j_{1} \cdots j_{d}}\right\|_{\mathbb{R}^{p}}^{2} \|\right. \\
&\left.\cdot \mathbf{1}\left\{\left\|\mathscr{E}_{j_{1} \cdots j_{d}}\right\|_{\mathbb{R}^{p}} \geq \frac{\epsilon \sqrt{n_{1} \cdots n_{d}}}{(r M)\left(d_{p} \Gamma_{f}^{2}\right)}\right\}\right) \quad \forall \epsilon>0,
\end{aligned}
$$

in which by the well-known bounded convergence theorem (cf. Athreya and Lahiri [28], pp. 57-58) the last term converges to zero. Thus the Lindeberg condition is satisfied. Therefore by the Lindeberg-Levy multivariate central limit theorem studied, for example, in van der Vaart [29], pp. 16, it can be concluded that $\sum_{k=1}^{r} c_{k} \mathscr{E}_{n_{1} \cdots n_{d}}\left(A_{k}\right)$ converges in distribution to $\sum_{k=1}^{r} c_{k} U\left(A_{k}\right)$, where $\sum_{k=1}^{r} c_{k} U\left(A_{k}\right)$ has the $p$-variate normal distribution with mean zero and the covariance given by (29).

The tightness of $\mathscr{Z}_{n_{1} \cdots n_{d}}$ can be shown as follows. By the definition $\mathscr{Z}_{n_{1} \cdots n_{d}}$ can also be expressed as

$$
\begin{aligned}
& \mathscr{Z}_{n_{1} \cdots n_{d}} \\
& \quad=\boldsymbol{\Sigma}^{-1 / 2} \operatorname{pr}_{\prod_{i=1}^{p} \mathbf{w}_{i, n_{1} \cdots n_{d} \mathscr{H}_{B}}} \boldsymbol{\Sigma}^{1 / 2} \boldsymbol{\Sigma}^{-1 / 2} \mathbf{V}_{n_{1} \cdots n_{d}}\left(\mathscr{E}_{n_{1} \cdots n_{d}}\right) .
\end{aligned}
$$


Since $\Sigma^{-1 / 2} \mathbf{V}_{n_{1} \cdots n_{d}}\left(\mathscr{E}_{n_{1} \cdots n_{d}}\right)$ is tight, hence by recalling Lemma 1 in Billingsley [19], pp. 38-40, it is sufficient to show that the

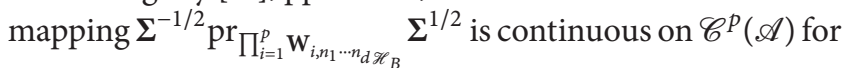
every $n_{1} \geq 1, \ldots, n_{d} \geq 1$. Proposition C.4 finishes the proof.

Corollary 2. By Theorem 1 and the well-known continuous mapping theorem (cf. Theorem 5.1 in Billingsley [19]) the distribution of the statistics $K S_{n_{1} \cdots n_{d}, \mathscr{A}}$ and $C v M_{n_{1} \cdots n_{d}, \mathscr{A}}$ can be approximated, respectively, by those of

$$
\begin{gathered}
K S:=\sup _{A \in \mathscr{A}}\left\|\left(\mathbf{B}_{p}-p r_{\prod_{i=1}^{p} \mathbf{w}_{i \mathscr{H}_{B}}}^{*} \mathbf{B}_{p}\right)(A)\right\|_{\mathbb{R}^{p}} \\
C v M:=\int_{\mathbf{I}^{d}}\left\|\left(\mathbf{B}_{p}-p r_{\prod_{i=1}^{p} \mathbf{w}_{i \mathscr{H}_{B}}}^{*} \mathbf{B}_{p}\right)(A)\right\|_{\mathbb{R}^{p}}^{2} d A .
\end{gathered}
$$

Let $\widetilde{t}_{1-\alpha}$ and $\widetilde{c}_{1-\alpha}$ be the $(1-\alpha)$ th quantile of the distributions of $K S$ and $C v M$, respectively. When $K S_{n_{1} \cdots n_{d}, \mathscr{A}}$ is used, $H_{0}$ will be rejected at level $\alpha$ if and only if $K S_{n_{1} \cdots n_{d}, \mathscr{A}} \geq \widetilde{t}_{1-\alpha}$. Likewise if CvM $M_{n_{1} \cdots n_{d}, \mathscr{A}}$ is used, then $H_{0}$ will be rejected at level $\alpha$ if and only if $C v M_{n_{1} \cdots n_{d}, \mathscr{A}} \geq \widetilde{c}_{1-\alpha}$.

\section{The Limit of $\mathbf{V}_{n_{1} \cdots n_{d}}\left(\mathbf{R}_{n_{1} \cdots n_{d}}\right)$ under $H_{1}$}

The test procedures derived above are consistent in the sense of Definition 11.1.3 in Lehmann and Romano [30]. That is, the probability of rejection of $H_{0}$ under the competing alternative converges to 1 . As an immediate consequence we cannot observe the performance of the tests when the model moves away from $H_{0}$. Therefore, to be able to investigate the behavior of the tests, we consider the localized model defined as follows:

$$
\mathbf{Z}_{n_{1} \cdots n_{d}}=\frac{1}{\sqrt{n_{1} \cdots n_{d}}} \mathbf{g}\left(\boldsymbol{\Xi}_{n_{1} \cdots n_{d}}\right)+\mathscr{E}_{n_{1} \cdots n_{d}} .
$$

When $H_{0}$ is true we get the similar least squares residuals as given in Section 1. Therefore, observing Model (35), the test problem will not be altered.
In the following theorem, we present the limit process of the $p$-dimensional set-indexed partial sums process of the residuals under $H_{1}$ associated with Model (35).

Theorem 3. For $i=1, \ldots, p$, let $\left\{f_{i 1}, \ldots, f_{i d_{i}}\right\}$ be an ONB of $\mathbf{W}_{i}$ with $\mathbf{W}_{i} \subseteq \mathbf{W}_{i+1}$, for $i=1, \ldots, p-1$. If $\mathbf{g}=\left(g_{i}\right)_{i=1}^{p} \in$ $\prod_{i=1}^{p} \operatorname{BVV}\left(\mathbf{I}^{d}\right)$ and $\left\{f_{i 1}, \ldots, f_{i d_{i}}\right\} \in \mathscr{C}\left(\mathbf{I}^{d}\right) \cap \mathrm{BV}_{H}\left(\mathbf{I}^{d}\right)$ (see Definition A.3 for the notion of $\mathrm{BVV}\left(\mathrm{I}^{d}\right)$ ), then, observing (35), we have under $H_{1}$ that

$$
\begin{aligned}
\Sigma^{-1 / 2} \mathbf{V}_{n_{1} \cdots n_{d}}\left(\mathbf{R}_{n_{1} \cdots n_{d}}\right) \stackrel{\mathscr{D}}{\longrightarrow} & \Sigma^{-1 / 2}\left(\mathbf{I}_{p}-p r_{\prod_{i=1}^{p} \mathbf{w}_{i \mathscr{H}_{B}}}^{*}\right) \mathbf{h}_{\mathbf{g}} \\
& +\mathbf{B}_{p, \mathbf{f}}^{H_{0}}
\end{aligned}
$$

where $\mathbf{h}_{\mathbf{g}}:=\left(h_{g_{i}}\right)_{i=1}^{p}: \mathscr{A} \rightarrow \mathscr{C}^{p}(\mathscr{A})$, with $h_{g_{i}}(A):=$ $\int_{A} g_{i}(\mathbf{t}) \lambda_{\mathbf{I}}^{d}(d \mathbf{t})$.

Proof. Considering the linearity of $\mathbf{V}_{n_{1} \cdots n_{d}}$ and Lemma C.2, when $H_{1}$ is true we have

$$
\begin{aligned}
& \boldsymbol{\Sigma}^{-1 / 2} \mathbf{V}_{n_{1} \cdots n_{d}}\left(\mathbf{R}_{n_{1} \cdots n_{d}}\right)=\Sigma^{-1 / 2} \frac{1}{\sqrt{n_{1} \cdots n_{d}}} \\
& \cdot \mathbf{V}_{n_{1} \cdots n_{d}}\left(\mathbf{g}\left(\boldsymbol{\Xi}_{n_{1} \cdots n_{d}}\right)\right)-\Sigma^{-1 / 2} \operatorname{pr}_{\prod_{i=1}^{p} \mathbf{w}_{i, n_{1} \cdots n_{d} \mathscr{C _ { B }}}} \\
& \cdot \frac{1}{\sqrt{n_{1} \cdots n_{d}}} \mathbf{V}_{n_{1} \cdots n_{d}}\left(\mathbf{g}\left(\boldsymbol{\Xi}_{n_{1} \cdots n_{d}}\right)\right) \\
& +\boldsymbol{\Sigma}^{-1 / 2} \mathbf{V}_{n_{1} \cdots n_{d}}\left(\mathscr{E}_{n_{1} \cdots n_{d}}\right) \\
& -\boldsymbol{\Sigma}^{-1 / 2} \mathrm{pr}_{\prod_{i=1}^{p} \mathbf{w}_{i, n_{1} \cdots n_{d} \mathscr{H}_{B}}} \mathbf{V}_{n_{1} \cdots n_{d}}\left(\mathscr{E}_{n_{1} \cdots n_{d}}\right)
\end{aligned}
$$

Since, for $i=1, \ldots, p, g_{i}$ is in $\operatorname{BVV}\left(\mathbf{I}^{d}\right)$, it can be shown that $\left(1 / \sqrt{n_{1} \cdots n_{d}}\right) \mathbf{V}_{n_{1} \cdots n_{d}}\left(\mathbf{g}\left(\boldsymbol{\Xi}_{n_{1} \cdots n_{d}}\right)\right)(B)$ converges uniformly to $\mathbf{h}_{\mathrm{g}}(B)$, for every $B \in \mathscr{A}$. Also the last two terms on the righthand side of the preceding equation converge in distribution to $\mathbf{B}_{p, \mathbf{f}}^{H_{0}}$ by Theorem 1 . Thus to the rest we only need to show that $\mathrm{pr}_{\prod_{i=1}^{p} \mathbf{w}_{i, n_{1} \cdots n_{d} \mathscr{H}_{B}}}\left(1 / \sqrt{n_{1} \cdots n_{d}}\right) \mathbf{V}_{n_{1} \cdots n_{d}}\left(\mathbf{g}_{n_{1} \cdots n_{d}}\right)$ converges to

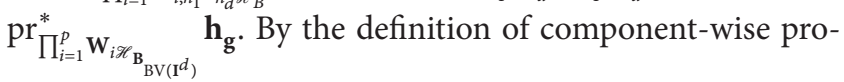
jection we have

$$
\begin{aligned}
\operatorname{pr}_{\prod_{i=1}}^{p} \mathbf{w}_{i, n_{1} \cdots n_{d} \mathscr{H}_{B}} \frac{1}{\sqrt{n_{1} \cdots n_{d}}} \mathbf{V}_{n_{1} \cdots n_{d}}\left(\mathbf{g}\left(\boldsymbol{\Xi}_{n_{1} \cdots n_{d}}\right)\right)=\left(\operatorname{pr}_{\mathbf{W}_{i, n_{1} \cdots n_{d} \mathscr{H} B}} \frac{1}{\sqrt{n_{1} \cdots n_{d}}} \mathbf{V}_{n_{1} \cdots n_{d}}^{(i)}\left(\mathbf{g}_{i}\left(\boldsymbol{\Xi}_{n_{1} \cdots n_{d}}\right)\right)\right)_{i=1}^{p} \\
=\left(\sum_{j=1}^{d_{i}}\left(\int_{\mathbf{I}^{d}}^{(R)} \widetilde{s}_{i j}^{\left(n_{1} \cdots n_{d}\right)}(\mathbf{t}) d \frac{1}{\sqrt{n_{1} \cdots n_{d}}} \mathbf{V}_{n_{1} \cdots n_{d}}^{(i)}\left(\mathbf{g}_{i}\left(\boldsymbol{\Xi}_{n_{1} \cdots n_{d}}\right)\right)(\mathbf{t})\right) h_{\widetilde{s}_{i j}\left(n_{1} \cdots n_{d}\right)}\right)_{i=1}^{p} \\
\quad=\left(\sum_{j=1}^{d_{i}}\left(\frac{1}{n_{1} \cdots n_{d}} \sum_{j_{1}=1}^{n_{1}} \cdots \sum_{j_{d}=1}^{n_{d}} \widetilde{s}_{i j}^{\left(n_{1} \cdots n_{d}\right)}\left(\frac{j_{1}}{n_{1}}, \ldots, \frac{j_{d}}{n_{d}}\right) g_{i}\left(\frac{j_{1}}{n_{1}}, \ldots, \frac{j_{d}}{n_{d}}\right)\right) h_{\widetilde{s}_{i j}\left(n_{1} \cdots n_{d}\right)}\right)_{i=1}^{p} .
\end{aligned}
$$

The right-hand side of the last expression is obtained directly from the definition of the ordinary partial sums (11) and the definition of the Riemann-Stieltjes integral on $\mathbf{I}^{d}$. Since $\widetilde{s}_{i j}^{\left(n_{1} \cdots n_{d}\right)}$ converges uniformly to $f_{i j}$ and $g_{i}$ has bounded variation 
on $\mathbf{I}^{d}$ for all $i$ and $j$, then the last expression clearly converges component-wise to $\left(\sum_{j=1}^{d_{i}}\left(\int_{\mathbf{I}^{d}}^{(R)} f_{i j}(\mathbf{t}) d h_{g_{i}}(\mathbf{t})\right) h_{f_{i j}}\right)_{i=1}^{p}$, which can be written as $\operatorname{pr}_{\prod_{i=1}^{p} \mathbf{w}_{i \mathscr{\ell}}}^{*} \mathbf{h}_{\mathbf{g}}$. We are done.

Corollary 4. By Theorem 3 the power function of the KS and CvM tests at a level $\alpha$ can now be approximated by computing the probabilities of the form

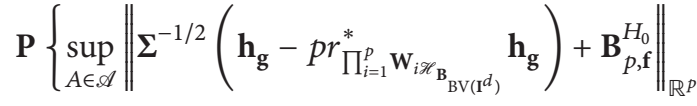

$$
\begin{aligned}
& \left.\geq \tilde{t}_{1-\alpha}\right\}
\end{aligned}
$$

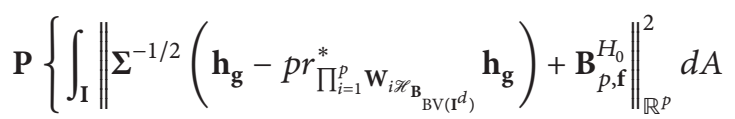

$$
\begin{aligned}
& \left.\geq \tilde{c}_{1-\alpha}\right\}
\end{aligned}
$$

for a fixed $\mathbf{g} \in \prod_{i=1}^{p} \operatorname{BVV}\left(\mathbf{I}^{d}\right)$, respectively. In Section 5 we investigate the empirical power functions of the KS and CvM tests by simulation.

\section{Estimating the Population Covariance Matrix}

If the covariance matrix $\boldsymbol{\Sigma}$ is unknown, as it usually is, it is impossible to use $\mathrm{KS}_{n_{1} \cdots n_{d} ; \mathscr{A}}$ and $\mathrm{CvM}_{n_{1} \cdots n_{d} ; \mathscr{A}}$ in practice. What we propose to do is to employ a consistent estimate of $\boldsymbol{\Sigma}$. We need some further notations for expressing the residuals of the model. For $i=1, \ldots, p$, let $Z_{i}^{\left(n_{1} \cdots n_{d}\right)}, g_{i}^{\left(n_{1} \cdots n_{d}\right)}$, and $\varepsilon_{i}^{\left(n_{1} \cdots n_{d}\right)}$ be $\left(n_{1} \cdots n_{d}\right)$-dimensional column vectors defined by

$$
\begin{aligned}
Z_{i}^{\left(n_{1} \cdots n_{d}\right)}:=\left(Z_{i}\left(\frac{j_{1}}{n_{1}}, \ldots, \frac{j_{d}}{n_{d}}\right)\right)_{j_{1}=1, \ldots, j_{d}=1}^{n_{1}, \ldots, n_{d}} \in \mathbb{R}^{n_{1} \cdots n_{d}} \\
g_{i}^{\left(n_{1} \cdots n_{d}\right)}:=\left(g_{i}\left(\frac{j_{1}}{n_{1}}, \ldots, \frac{j_{d}}{n_{d}}\right)\right)_{j_{1}=1, \ldots, j_{d}=1}^{n_{1}, \ldots, n_{d}} \in \mathbb{R}^{n_{1} \cdots n_{d}} \\
\varepsilon_{i}^{\left(n_{1} \cdots n_{d}\right)}:=\left(\varepsilon_{i}\left(\frac{j_{1}}{n_{1}}, \ldots, \frac{j_{d}}{n_{d}}\right)\right)_{j_{1}=1, \ldots, j_{d}=1}^{n_{1}, \ldots, n_{d}} \in \mathbb{R}^{n_{1} \cdots n_{d}} .
\end{aligned}
$$

Furthermore, let $\mathbf{Z}^{\left(n_{1} \cdots n_{d}\right)}, \mathbf{g}^{\left(n_{1} \cdots n_{d}\right)}$, and $\mathscr{E}^{\left(n_{1} \cdots n_{d}\right)}$ be $\left(n_{1} \cdots n_{d}\right) \times$ $p$-dimensional matrices whose $i$ th column is given, respectively, by the column vectors $Z_{i}^{\left(n_{1} \cdots n_{d}\right)}, g_{i}^{\left(n_{1} \cdots n_{d}\right)}$, and $\varepsilon_{i}^{\left(n_{1} \cdots n_{d}\right)}$, $i=1, \ldots, p$. Then Model (35) can also be represented as follows:

$$
\mathbf{Z}^{\left(n_{1} \cdots n_{d}\right)}=\frac{1}{\sqrt{n_{1} \cdots n_{d}}} \mathbf{g}^{\left(n_{1} \cdots n_{d}\right)}+\mathscr{E}^{\left(n_{1} \cdots n_{d}\right)},
$$

where, for $u, v=1, \ldots, p, \operatorname{Cov}\left(\varepsilon_{u}^{\left(n_{1} \cdots n_{d}\right)}, \varepsilon_{v}^{\left(n_{1} \cdots n_{d}\right)}\right)=\sigma_{u v} \mathbf{I}_{n_{1} \cdots n_{d}}$, with $\mathbf{I}_{n_{1} \cdots n_{d}}$ being the identity matrix in $\mathbb{R}^{\left(n_{1} \cdots n_{d}\right) \times\left(n_{1} \cdots n_{d}\right)}$.

Associated with the subspace $\mathbf{W}_{i, n_{1} \cdots n_{d}}$ we define the design matrix $\mathbf{X}_{i}^{\left(n_{1} \cdots n_{d}\right)}$ as an element of $\mathbb{R}^{\left(n_{1} \cdots n_{d}\right) \times d_{i}}$ whose $u$ th column is given by the $\left(n_{1} \cdots n_{d}\right)$-dimensional column vector:

$$
\begin{aligned}
&\left(f_{\text {iu }}\left(\frac{j_{1}}{n_{1}}, \ldots, \frac{j_{d}}{n_{d}}\right)\right)_{j_{1}=1, \ldots, j_{d}=1}^{n_{1}, \ldots, n_{d}} \in \mathbb{R}^{n_{1} \cdots n_{d}}, \\
& u=1, \ldots, d_{i}, i=1, \ldots, p .
\end{aligned}
$$

We denote the column space of $\mathbf{X}_{i}^{\left(n_{1} \cdots n_{d}\right)}$ by $\mathscr{C}\left(\mathbf{X}_{i}^{\left(n_{1} \cdots n_{d}\right)}\right) \subset$ $\mathbb{R}^{\left(n_{1} \cdots n_{d}\right) \times d_{i}}$ for the sake of brevity. We also define the columnwise projection of any matrix $\mathbf{U}^{\left(n_{1} \cdots n_{d}\right)}:=\left(U_{1}^{\left(n_{1} \cdots n_{d}\right)}, \ldots\right.$, $\left.U_{p}^{\left(n_{1} \cdots n_{d}\right)}\right)$ in $\mathbb{R}^{n_{1} \cdots n_{d} \times p}$ into the product space $\prod_{i=1}^{p} \mathscr{C}\left(\mathbf{X}_{i}^{\left(n_{1} \cdots n_{d}\right)}\right)$ by

$$
\begin{aligned}
& \operatorname{pr}_{\prod_{i=1}^{p} \mathscr{C}\left(\mathbf{X}_{i}^{\left(n_{1} \cdots n_{d}\right)}\right)} \mathbf{U}^{\left(n_{1} \cdots n_{d}\right)} \\
& \quad:=\left(\operatorname{pr}_{\mathscr{C}\left(\mathbf{X}_{1}^{\left(n_{1} \cdots n_{d}\right)}\right)} U_{1}^{\left(n_{1} \cdots n_{d}\right)}, \ldots, \operatorname{pr}_{\mathscr{C}\left(\mathbf{X}_{p}^{\left(n_{1} \cdots n_{d}\right)}\right)} U_{p}^{\left(n_{1} \cdots n_{d}\right)}\right) .
\end{aligned}
$$

A reasonable estimator of the covariance matrix $\Sigma$ is denoted by $\widehat{\Sigma}_{n_{1} \cdots n_{d}}$, defined by

$$
\begin{aligned}
\widehat{\Sigma}_{n_{1} \cdots n_{d}}:= & \frac{1}{n_{1} \cdots n_{d}}\left(\operatorname{pr}_{\prod_{i=1}^{p} \mathscr{E}\left(\mathbf{X}_{i}^{\left(n_{1} \cdots n_{d}\right)}\right)_{\perp}} \mathbf{Z}^{\left(n_{1} \cdots n_{d}\right)}\right)^{\top} \\
& \cdot\left(\operatorname{pr}_{\prod_{i=1}^{p} \mathscr{C}\left(\mathbf{X}_{i}^{\left(n_{1} \cdots n_{d}\right)}\right)^{\perp}} \mathbf{Z}^{\left(n_{1} \cdots n_{d}\right)}\right)
\end{aligned}
$$

where

$$
\operatorname{pr}_{\prod_{i=1}^{p} \mathscr{C}\left(\mathbf{X}_{i}^{\left(n_{1} \cdots n_{d}\right)}\right)^{\perp}}:=\mathbf{I}_{n_{1} \cdots n_{d}}-\operatorname{pr}_{\prod_{i=1}^{p} \mathscr{C}\left(\mathbf{X}_{i}^{\left(n_{1} \cdots n_{d}\right)}\right)}
$$

constitutes the component-wise orthogonal projector into the orthogonal complement of the product space $\prod_{i=1}^{p} \mathscr{C}\left(\mathbf{X}_{i}^{\left(n_{1} \cdots n_{d}\right)}\right)$.

Zellner [1] and Arnold [22] investigated the consistency of $\widehat{\Sigma}_{n_{1} \cdots n_{d}}$ toward $\boldsymbol{\Sigma}$ in the case of the multivariate regression model with $\mathbf{W}_{1}=\cdots=\mathbf{W}_{p}$. Some difficulties appear when the situation is extended to the case of $\mathbf{W}_{1} \neq \cdots \neq \mathbf{W}_{p}$, since it involves the problem of finding the limit of matrices with the components given by inner products of two vectors.

Theorem 5. Suppose the localized model (41) is observed. If $H_{0}$ is true, then, under the conditions of Theorem 1, we have $\widehat{\Sigma}_{n_{1} \cdots n_{d}} \stackrel{\mathscr{P}}{\rightarrow} \Sigma$.

Proof. If $H_{0}$ is true, it can be easily shown that

$$
\begin{aligned}
\left(n_{1} \cdots n_{d}\right) \widehat{\Sigma}_{n_{1} \cdots n_{d}}= & \left(\operatorname{pr}_{\prod_{i=1}^{p} \mathscr{C}\left(\mathbf{X}_{i}^{\left(n_{1} \cdots n_{d}\right)}\right)^{\perp}} \mathscr{E}^{\left(n_{1} \cdots n_{d}\right)}\right)^{\top} \\
& \cdot\left(\operatorname{pr}_{\prod_{i=1}^{p} \mathscr{E}\left(\mathbf{X}_{i}^{\left(n_{1} \cdots n_{d}\right)}\right)^{\perp}} \mathscr{E}^{\left(n_{1} \cdots n_{d}\right)}\right) .
\end{aligned}
$$


For technical reason we assume without lost of generality that $\mathbf{X}_{i}^{\left(n_{1} \cdots n_{d}\right)}$ is an orthogonal matrix, for $i=1, \ldots, p$. Hence we further get the representation

$$
\begin{aligned}
& \left(n_{1} \cdots n_{d}\right) \widehat{\Sigma}_{n_{1} \cdots n_{d}} \\
& =\left(\varepsilon_{u}^{\left(n_{1} \cdots n_{d}\right)^{\top}} \varepsilon_{v}^{\left(n_{1} \cdots n_{d}\right)}\right)_{u, v=1}^{p, p} \\
& -\left(\left(\mathbf{X}_{v}^{\left(n_{1} \cdots n_{d}\right) \top} \varepsilon_{u}^{\left(n_{1} \cdots n_{d}\right)}\right)^{\top}\left(\mathbf{X}_{v}^{\left(n_{1} \cdots n_{d}\right) \top} \varepsilon_{v}^{\left(n_{1} \cdots n_{d}\right)}\right)\right)_{u, v=1}^{p, p} \\
& -\left(\left(\mathbf{X}_{u}^{\left(n_{1} \cdots n_{d}\right) \top} \varepsilon_{u}^{\left(n_{1} \cdots n_{d}\right)}\right)^{\top}\left(\mathbf{X}_{u}^{n_{1} \cdots n_{d} \top} \varepsilon_{v}^{\left(n_{1} \cdots n_{d}\right)}\right)\right)_{u, v=1}^{p, p} \\
& +\left(\left(\mathbf{X}_{u}^{\left(n_{1} \cdots n_{d}\right) \top} \varepsilon_{u}^{\left(n_{1} \cdots n_{d}\right)}\right)^{\top}\left(\mathbf{X}_{v}^{\left(n_{1} \cdots n_{d}\right) \top} \varepsilon_{v}^{\left(n_{1} \cdots n_{d}\right)}\right)\right)_{u, v=1}^{p, p} .
\end{aligned}
$$

Since $\left(\varepsilon_{u}\left(j_{1} / n_{1}, \ldots, j_{d} / n_{d}\right) \varepsilon_{v}\left(j_{1} / n_{1}, \ldots, j_{d} / n_{d}\right)\right)_{u, v=1}^{p, p}$ are independent and identically distributed random matrices with mean $\Sigma$, by the well-known weak law of large numbers, we get

$$
\begin{gathered}
\frac{1}{n_{1} \cdots n_{d}} \sum_{j_{1}=1}^{n_{1}} \ldots \sum_{j_{d}=1}^{n_{d}}\left(\varepsilon_{u}\left(\frac{j_{1}}{n_{1}}, \ldots, \frac{j_{d}}{n_{d}}\right)\right. \\
\left.\cdot \varepsilon_{v}\left(\frac{j_{1}}{n_{1}}, \ldots, \frac{j_{d}}{n_{d}}\right)\right)_{u, v=1}^{p, p} \stackrel{\mathscr{P}}{\longrightarrow} \Sigma .
\end{gathered}
$$

Note that in the practice we consider the polynomial regression model. Hence, for every $v=1, \ldots, p$, the design matrix $\mathbf{X}_{v}^{\left(n_{1} \cdots n_{d}\right)}$ satisfies the so-called Huber condition (cf. Pruscha [31], pp. 115-117). By this reason, for the rest of the terms, we can immediately apply the technique proposed in Arnold [22] to show $\mathbf{X}_{v}^{\left(n_{1} \cdots n_{d}\right) \top} \varepsilon_{u}^{\left(n_{1} \cdots n_{d}\right)} \stackrel{\mathscr{D}}{\rightarrow} N_{d_{v}}\left(\mathbf{0}, \sigma_{u u} \mathbf{I}_{d_{v}}\right)$, for all $u, v=$ $1, \ldots, p$. Therefore, we finally get the following componentwise convergence:

$$
\begin{aligned}
& \frac{1}{n_{1} \cdots n_{d}}\left(\left(\mathbf{X}_{v}^{\left(n_{1} \cdots n_{d}\right) \top} \varepsilon_{u}^{\left(n_{1} \cdots n_{d}\right)}\right)^{\top}\right. \\
& \left.\cdot\left(\mathbf{X}_{v}^{\left(n_{1} \cdots n_{d}\right) \top} \varepsilon_{v}^{\left(n_{1} \cdots n_{d}\right)}\right)\right)_{u, v=1}^{p, p} \stackrel{\mathscr{P}}{\longrightarrow} \mathbf{O}_{p \times p} \\
& \frac{1}{n_{1} \cdots n_{d}}\left(\left(\mathbf{X}_{u}^{\left(n_{1} \cdots n_{d}\right) \top} \varepsilon_{u}^{\left(n_{1} \cdots n_{d}\right)}\right)^{\top}\right. \\
& \left.\cdot\left(\mathbf{X}_{u}^{\left(n_{1} \cdots n_{d}\right) \top} \varepsilon_{v}^{\left(n_{1} \cdots n_{d}\right)}\right)\right)_{u, v=1}^{p, p} \stackrel{\mathscr{P}}{\longrightarrow} \mathbf{O}_{p \times p} \\
& \frac{1}{n_{1} \cdots n_{d}}\left(\left(\mathbf{X}_{u}^{\left(n_{1} \cdots n_{d}\right) \top} \varepsilon_{u}^{\left(n_{1} \cdots n_{d}\right)}\right)^{\top}\right. \\
& \left.\cdot\left(\mathbf{X}_{v}^{\left(n_{1} \cdots n_{d}\right) \top} \varepsilon_{v}^{\left(n_{1} \cdots n_{d}\right)}\right)\right)_{u, v=1}^{p, p} \stackrel{\mathscr{P}}{\longrightarrow} \mathbf{O}_{p \times p},
\end{aligned}
$$

where $\mathbf{O}_{p \times p}$ is the $p \times p$-zero matrix.

$$
\text { Remark 6. Since } \widehat{\boldsymbol{\Sigma}}_{n_{1} \cdots n_{d}}^{-1 / 2} \mathbf{V}_{n_{1} \cdots n_{d}}\left(\mathbf{R}_{n_{1} \cdots n_{d}}\right)=
$$
$\widehat{\boldsymbol{\Sigma}}_{n_{1} \cdots n_{d}}^{-1 / 2} \Sigma^{1 / 2} \boldsymbol{\Sigma}^{-1 / 2} \mathbf{V}_{n_{1} \cdots n_{d}}\left(\mathbf{R}_{n_{1} \cdots n_{d}}\right)$, without altering the convergence result presented in Theorem 1 , the population variance-covariance matrix $\Sigma$ can be directly replaced by the consistence estimator $\widehat{\Sigma}_{n_{1} \cdots n_{d}}$.

\section{Calibration of the Tests}

The limits of the test statistics are not distribution-free and we need therefore calibration for the distribution of the statistical tests. For the calibration we adapted the idea of residual based bootstrap for multivariate regression studied in Shao and $\mathrm{Tu}$ [32] for approximating the distributions of $\mathrm{KS}_{n_{1} \cdots n_{d}, \mathscr{A}}$ and $\mathrm{CvM}_{n_{1} \cdots n_{d}, \mathscr{A}}$.

For fixed $n_{1}, \ldots, n_{d}$, let $F_{n_{1} \cdots n_{d}}$ be the empirical distribution function of the vectors of least squares residuals $\left\{\mathbf{r}_{j_{1} \cdots j_{d}}-\right.$ $\left.\overline{\mathbf{R}}_{n_{1} \cdots n_{d}}: 1 \leq j_{k} \leq n_{k}, k=1, \ldots, d\right\}$ centered at zero vector, where $\overline{\mathbf{R}}_{n_{1} \cdots n_{d}}:=\left(1 /\left(n_{1} \cdots n_{d}\right)\right) \sum_{j_{1}=1}^{n_{1}} \cdots \sum_{j_{d}=1}^{n_{d}} \mathbf{r}_{j_{1} \cdots j_{d}}$. Let $\mathscr{E}_{n_{1} \cdots n_{d}}^{*}:=\left(\mathscr{E}_{j_{1} \cdots j_{d}}^{*}\right)_{j_{1}=1, \ldots, j_{d}=1}^{n_{1}, \ldots, n_{d}}$ be an array of independent and identically distributed random vectors sampled from $F_{n_{1} \cdots n_{d}}$ and let $\widehat{\mathbf{g}}\left(\Xi_{n_{1} \cdots n_{d}}\right)$ be the ordinary LSE of $\mathbf{g}\left(\Xi_{n_{1} \cdots n_{d}}\right)$, where $\widehat{\mathbf{g}}\left(\Xi_{n_{1} \cdots n_{d}}\right)=\mathrm{pr}_{\prod_{i=1}^{p} \mathbf{w}_{i}} \mathbf{Z}_{n_{1} \cdots n_{d}}$. Then we generate the array of $p$-dimensional bootstrap observations which is denoted in this paper by $\mathbf{Z}_{n_{1} \cdots n_{d}}^{*}:=\left(\mathbf{Z}_{j_{1} \cdots j_{d}}^{*}\right)_{j_{1}=1, \ldots, j_{d}=1}^{n_{1}, \ldots, n_{d}}$ through the model:

$$
\mathbf{Z}_{n_{1} \cdots n_{d}}^{*}=\operatorname{pr}_{\prod_{i=1}^{p} \mathbf{w}_{i}} \mathbf{Z}_{n_{1} \cdots n_{d}}+\mathscr{E}_{n_{1} \cdots n_{d}}^{*} .
$$

Based on this model we get the array of $p$-dimensional bootstrap least squares residuals which is given by the component-wise projection of the bootstrap observations:

$$
\begin{aligned}
\mathbf{R}_{n_{1} \cdots n_{d}}^{*} & :=\left(\mathbf{r}_{j_{1} \cdots j_{d}}^{*}\right)_{j_{1}=1, \ldots, j_{d}=1}^{n_{1}, \ldots, n_{d}} \\
& =\mathbf{Z}_{n_{1} \cdots n_{d}}^{*}-\operatorname{pr}_{\prod_{i=1}^{p} \mathbf{w}_{i}} \mathbf{Z}_{n_{1} \cdots n_{d}}^{*} .
\end{aligned}
$$

Hence, the bootstrap analog of $\mathrm{KS}_{n_{1} \cdots n_{d}, \mathscr{A}}$ and $\mathrm{CvM}_{n_{1} \cdots n_{d}, \mathscr{A}}$ is

$$
\begin{aligned}
& \mathrm{KS}_{n_{1} \cdots n_{d}, \mathscr{A}}^{*}:=\sup _{A \in \mathscr{A}}\left\|\widehat{\Sigma}_{n_{1} \cdots n_{d}}^{*-1 / 2} \mathbf{V}_{n_{1} \cdots n_{d}}\left(\mathbf{R}_{n_{1} \cdots n_{d}}^{*}\right)(A)\right\|_{\mathbb{R}^{p}} \\
& \mathrm{CvM}_{n_{1} \cdots n_{d}, \mathscr{A}}^{*} \\
& \quad:=\frac{1}{n_{1} \cdots n_{d}} \sum_{A \in \mathscr{A}}\left\|\widehat{\Sigma}_{n_{1} \cdots n_{d}}^{*-1 / 2} \mathbf{V}_{n_{1} \cdots n_{d}}\left(\mathbf{R}_{n_{1} \cdots n_{d}}^{*}\right)(A)\right\|_{\mathbb{R}^{p}}^{2},
\end{aligned}
$$

where

$$
\begin{aligned}
\widehat{\Sigma}_{n_{1} \cdots n_{d}}^{*}:= & \frac{1}{n_{1} \cdots n_{d}}\left(\operatorname{pr}_{\prod_{i=1}^{p} \mathscr{C}\left(\mathbf{X}_{i}^{\left(n_{1} \cdots n_{d}\right)}\right)^{\perp}} \mathbf{Z}^{*\left(n_{1} \cdots n_{d}\right)}\right)^{\top} \\
& \cdot\left(\operatorname{pr}_{\prod_{i=1}^{p} \mathscr{C}\left(\mathbf{X}_{i}^{\left(n_{1} \cdots n_{d}\right)}\right)^{\perp}} \mathbf{Z}^{*\left(n_{1} \cdots n_{d}\right)}\right) .
\end{aligned}
$$

The question regarding the consistency of the bootstrap approximation of the $p$-dimensional processes $\widehat{\boldsymbol{\Sigma}}_{n_{1} \cdots n_{d}}^{*-1 / 2} \mathbf{V}_{n_{1} \cdots n_{d}}\left(\mathbf{R}_{n_{1} \cdots n_{d}}^{*}\right)(A)$ for $\widehat{\boldsymbol{\Sigma}}_{n_{1} \cdots n_{d}}^{-1 / 2} \mathbf{V}_{n_{1} \cdots n_{d}}\left(\mathbf{R}_{n_{1} \cdots n_{d}}\right)(A)$ is summarized in the following theorem. 
Theorem 7. Let $\left\{f_{i 1}, \ldots, f_{i d_{i}}\right\}$ be an ONB of $\mathbf{W}_{i}$, for $i=$ $1, \ldots, p$. Suppose the conditions of Theorem 1 are fulfilled. Then under $\mathrm{H}_{0}$ it holds that

$$
\Sigma^{*-1 / 2} \mathbf{V}_{n_{1} \cdots n_{d}}\left(\mathbf{R}_{n_{1} \cdots n_{d}}^{*}\right) \stackrel{\mathscr{D}}{\longrightarrow} \mathbf{B}_{p}-\operatorname{pr}_{\prod_{i=1}^{p} \mathbf{w}_{i \mathscr{H}_{B}}}^{*} \mathbf{B}_{p}
$$

where $\operatorname{pr}_{\prod_{i=1}^{p} \mathbf{w}_{i \mathscr{H}_{B}}}^{*} \mathbf{B}_{p}$ is defined in Theorem 1.

Proof. We notice that $\left\{\mathscr{E}_{j_{1} \cdots j_{d}}^{*}: 1 \leq j_{k} \leq n_{k}, k=\right.$ $1, \ldots, d\}$ are independent and identically distributed with $E_{*}\left(\mathscr{E}_{1 \cdots 1}^{*}\right)=\mathbf{0}$ and $\operatorname{Cov}_{*}\left(\mathscr{E}_{1 \cdots 1}^{*}\right)=\widehat{\boldsymbol{\Sigma}}_{n_{1} \cdots n_{d}}-\overline{\mathbf{R}}_{n_{1} \cdots n_{d}} \overline{\mathbf{R}}_{n_{1} \cdots n_{d}}^{\top}$. Hence, the invariance principle implies that $\left(\widehat{\Sigma}_{n_{1} \cdots n_{d}}-\right.$ $\left.\overline{\mathbf{R}}_{n_{1} \cdots n_{d}} \overline{\mathbf{R}}_{n_{1} \cdots n_{d}}^{\top}\right)^{-1 / 2} \mathbf{V}_{n_{1} \cdots n_{d}}\left(\mathscr{E}_{n_{1} \cdots n_{d}}^{*}\right)$ converges in distribution to $\mathbf{B}_{p}$. Hence under $H_{0}$ we have $\mathbf{R}_{n_{1} \cdots n_{d}}^{*}=\mathscr{E}_{n_{1} \cdots n_{d}}^{*}-\operatorname{pr}_{\prod_{i=1}^{p} \mathbf{w}_{i}} \mathscr{E}_{n_{1} \cdots n_{d}}^{*}$ and $\Sigma_{n_{1} \cdots n_{d}}^{*-1 / 2}$ can be written as

$$
\begin{aligned}
\Sigma_{n_{1} \cdots n_{d}}^{*-1 / 2}= & \Sigma_{n_{1} \cdots n_{d}}^{*-1 / 2}\left(\widehat{\boldsymbol{\Sigma}}_{n_{1} \cdots n_{d}}-\overline{\mathbf{R}}_{n_{1} \cdots n_{d}} \overline{\mathbf{R}}_{n_{1} \cdots n_{d}}^{\top}\right)^{1 / 2} \\
& \cdot\left(\widehat{\boldsymbol{\Sigma}}_{n_{1} \cdots n_{d}}-\overline{\mathbf{R}}_{n_{1} \cdots n_{d}} \overline{\mathbf{R}}_{n_{1} \cdots n_{d}}^{\top}\right)^{-1 / 2}
\end{aligned}
$$

where it can be shown easily that

$$
\begin{aligned}
\overline{\mathbf{R}}_{n_{1} \cdots n_{d}} \overline{\mathbf{R}}_{n_{1} \cdots n_{d}}^{\top} & =o_{\mathscr{P}}(1), \\
E_{*}\left(\widehat{\Sigma}_{n_{1} \cdots n_{d}}^{*}\right) & =\widehat{\Sigma}_{n_{1} \cdots n_{d}}+o_{\mathscr{P}}(1),
\end{aligned}
$$

with $o_{\mathscr{P}}(1)$ being the collection of the terms converging in probability to $\mathbf{O}_{p \times p}$. Then by recalling Theorem 5 and the linearity of $\mathbf{V}_{n_{1} \cdots n_{d}}$ we only need to show that

$$
\begin{aligned}
& \left(\widehat{\boldsymbol{\Sigma}}_{n_{1} \cdots n_{d}}-\overline{\mathbf{R}}_{n_{1} \cdots n_{d}} \overline{\mathbf{R}}_{n_{1} \cdots n_{d}}^{\top}\right)^{-1 / 2} \mathbf{V}_{n_{1} \cdots n_{d}}\left(\operatorname{pr}_{\prod_{i=1}^{p} \mathbf{w}_{i}} \mathscr{E}_{n_{1} \cdots n_{d}}^{*}\right) \stackrel{\mathscr{D}}{\longrightarrow} \\
& \operatorname{pr}_{\prod_{i=1}^{*} \mathbf{w}_{i \mathscr{K}_{B}}}^{*} \mathbf{B}_{p} .
\end{aligned}
$$

The proof is established by imitating the steps of proving convergence result of Theorem 1 . We are done.

\section{Simulation Study}

In this section, we report on a simulation study designed to investigate the finite sample size behavior of the KS and CvM tests. We simulate a multivariate model with four components defined on the unit rectangle $\mathbf{I}^{2}$. The hypothesis under study is $H_{0}: \mathbf{g} \in \prod_{i=1}^{4} \mathbf{W}_{i}$ against $H_{1}: \mathbf{g} \notin \prod_{i=1}^{4} \mathbf{W}_{i}$, where $\mathbf{W}_{1}:=$ $\left[f_{1}\right], \mathbf{W}_{2}:=\left[f_{1}, f_{2}, f_{3}\right], \mathbf{W}_{3}:=\left[f_{1}, f_{2}, f_{3}, f_{4}, f_{5}, f_{6}\right]$, and $\mathbf{W}_{4}:=\left[f_{1}, f_{2}, f_{3}, f_{4}, f_{5}, f_{6}, f_{7}\right]$. Thereby we define $f_{1}(t, s):=$ $1, f_{2}(t, s)=t, f_{3}(t, s)=s, f_{4}(t, s)=t^{2}, f_{5}(t, s)=$ $s^{2}, f_{6}(t, s)=t s$, and $f_{7}(t, s)=t^{3}$, for $(t, s) \in \mathbf{I}^{2}$. The samples are generated from a localized model $\mathrm{Y}\left(\ell / n_{1}, k / n_{2}\right)=$ $\left(1 / \sqrt{n_{1} n_{2}}\right) \mathbf{g}\left(\ell / n_{1}, k / n_{2}\right)+\mathscr{E}\left(\ell / n_{1}, k / n_{2}\right)$ under the experimental design given by $\Xi_{n_{1} \times n_{2}}=\left\{\left(\ell / n_{1}, k / n_{2}\right) \in \mathbf{I}^{2}: 1 \leq \ell \leq\right.$ $\left.n_{1}, 1 \leq k \leq n_{2}\right\}$, where $\mathbf{g}\left(\ell / n_{1}, k / n_{2}\right)$ is defined as

$$
\mathbf{g}\left(\frac{\ell}{n_{1}}, \frac{k}{n_{2}}\right):=\left(\begin{array}{c}
5+\rho \exp \left\{\frac{\ell}{n_{1}} \frac{k}{n_{2}}\right\} \\
10-5 \frac{\ell}{n_{1}}+10 \frac{k}{n_{2}}+\gamma \exp \left\{\frac{\ell}{n_{1}} \frac{k}{n_{2}}\right\} \\
10+20 \frac{\ell}{n_{1}}-25 \frac{k}{n_{2}}+10 \frac{\ell^{2}}{n_{1}^{2}}-5 \frac{k^{2}}{n_{2}^{2}}+20 \frac{\ell}{n_{1}} \frac{k}{n_{2}}+\delta \exp \left\{\frac{\ell}{n_{1}} \frac{k}{n_{2}}\right\} \\
30-30 \frac{\ell}{n_{1}}-5 \frac{k}{n_{2}}+20 \frac{\ell^{2}}{n_{1}^{2}}-15 \frac{k^{2}}{n_{2}^{2}}+10 \frac{\ell}{n_{1}} \frac{k}{n_{2}}+10 \frac{\ell^{3}}{n_{1}^{3}}+\kappa \exp \left\{\frac{\ell}{n_{1}} \frac{k}{n_{2}}\right\}
\end{array}\right),
$$

for constants $\rho, \gamma, \delta$, and $\kappa$ determined prior to the generation of the samples. For fixed $n_{1}$ and $n_{2}$ and $1 \leq \ell \leq n_{1}$ and $1 \leq$ $k \leq n_{2}$ the vector of random errors $\mathscr{E}\left(\ell / n_{1}, k / n_{2}\right)$ is generated independently from the 4 -variate normal distribution with mean zero and variance-covariance matrix given by

$$
\Sigma_{4 \times 4}:=\left(\begin{array}{cccc}
9 & 3 & -6 & 12 \\
3 & 26 & -7 & -11 \\
-6 & -7 & 9 & 7 \\
12 & -11 & 7 & 65
\end{array}\right)
$$

however, we assume in the computation that $\boldsymbol{\Sigma}_{4 \times 4}$ is unknown. It is therefore estimated using $\widehat{\boldsymbol{\Sigma}}_{n_{1} n_{2}}$ defined in Section 4. It is important to note that for computational reason we restricted the index set to the Vapnick Chervonenkis
Classes (VCC) of subsets of $\mathbf{I}^{2}$ which is given by the family of closed rectangle with the point $(0,0)$ as the essential point. That is, the family $\{[0, t] \times[0, s]: 0 \leq t, s \leq 1\}$.

When $\rho, \gamma, \delta$, and $\kappa$ are set simultaneously to zero then we get the samples which coincide to the model specified under $H_{0}$. Conversely, when at least one of them takes a nonzero value then we obtain the samples which can be regarded as from the alternative whose corresponding samples are generated by assigning nonzero values to either one of the constants, $\rho, \gamma, \delta$, and $\kappa$, or the combinations of them.

Table 1 presents the empirical probabilities of rejection of $H_{0}$ for $\alpha=0.05$ and some selected values of $\rho, \gamma, \delta$, and $\kappa$. The empirical powers of the KS and CvM tests are denoted by $\widehat{\alpha}_{\mathrm{KS}}$ and $\widehat{\alpha}_{\mathrm{CvM}}$, respectively. The notations $\widehat{\sigma}_{\mathrm{Ks}}$ and $\widehat{\sigma}_{\mathrm{CvM}}$ stand, respectively, for the standard deviation of the samples. The critical values of the statistics $\mathrm{KS}_{n_{1}, n_{2} ; \mathscr{A}}$ and $\mathrm{CvM}_{n_{1}, n_{2} ; \mathscr{A}}$ are 
TABLE 1: The empirical probabilities of rejection of the KS and CvM tests for several selected values of $\rho$, $\gamma, \delta$, and $\kappa$. The sample sizes are selected to $35 \times 40$ and $50 \times 60$. The simulation results are based on 10000 runs.

\begin{tabular}{|c|c|c|c|c|c|c|c|c|}
\hline Sample size & $\rho$ & $\gamma$ & $\delta$ & $\kappa$ & $\widehat{\alpha}_{\mathrm{KS}}$ & $\widehat{\sigma}_{\mathrm{KS}}$ & $\widehat{\alpha}_{\mathrm{CvM}}$ & $\widehat{\sigma}_{\mathrm{CvM}}$ \\
\hline \multirow{27}{*}{$35 \times 40$} & 0 & 0 & 0 & 0 & $4.90 \%$ & 1.31299 & $4.60 \%$ & 2.52127 \\
\hline & 10 & 0 & 0 & 0 & $10.60 \%$ & 1.52902 & $11.28 \%$ & 4.11538 \\
\hline & 20 & 0 & 0 & 0 & $32.80 \%$ & 1.71455 & $33.40 \%$ & 6.26419 \\
\hline & 40 & 0 & 0 & 0 & $88.64 \%$ & 1.86015 & $87.22 \%$ & 10.37546 \\
\hline & 0 & 50 & 0 & 0 & $5.03 \%$ & 1.28540 & $5.33 \%$ & 2.63926 \\
\hline & 0 & 200 & 0 & 0 & $4.43 \%$ & 1.22630 & $5.90 \%$ & 2.57881 \\
\hline & 0 & 300 & 0 & 0 & $4.60 \%$ & 1.18783 & $7.03 \%$ & 2.52180 \\
\hline & 0 & 500 & 0 & 0 & $4.63 \%$ & 0.90516 & $11.00 \%$ & 2.32439 \\
\hline & 0 & 0 & 50 & 0 & $4.83 \%$ & 1.27888 & $5.03 \%$ & 2.59347 \\
\hline & 0 & 0 & 100 & 0 & $5.17 \%$ & 1.29476 & $5.20 \%$ & 2.68005 \\
\hline & 0 & 0 & 200 & 0 & $6.03 \%$ & 1.28627 & $5.23 \%$ & 2.53931 \\
\hline & 0 & 0 & 500 & 0 & $8.23 \%$ & 1.27694 & $7.73 \%$ & 2.71275 \\
\hline & 0 & 0 & 0 & 50 & $5.00 \%$ & 1.27241 & $5.03 \%$ & 2.69462 \\
\hline & 0 & 0 & 0 & 100 & $4.77 \%$ & 1.23645 & $4.63 \%$ & 2.56912 \\
\hline & 0 & 0 & 0 & 300 & $4.77 \%$ & 1.27318 & $5.07 \%$ & 2.53470 \\
\hline & 0 & 0 & 0 & 500 & $4.97 \%$ & 1.26322 & $4.97 \%$ & 2.55223 \\
\hline & 10 & 50 & 0 & 0 & $11.34 \%$ & 1.55144 & $12.22 \%$ & 3.82300 \\
\hline & 10 & 100 & 0 & 0 & $11.12 \%$ & 1.49017 & $12.70 \%$ & 3.81009 \\
\hline & 10 & 200 & 0 & 0 & $11.20 \%$ & 1.52118 & $12.06 \%$ & 3.71303 \\
\hline & 10 & 500 & 0 & 0 & $12.46 \%$ & 1.10222 & $22.44 \%$ & 2.87591 \\
\hline & 20 & 50 & 50 & 50 & $32.78 \%$ & 1.76263 & $33.62 \%$ & 5.75683 \\
\hline & 20 & 50 & 100 & 50 & $31.80 \%$ & 1.71323 & $32.52 \%$ & 5.98971 \\
\hline & 20 & 50 & 200 & 50 & $28.16 \%$ & 1.68594 & $29.16 \%$ & 5.79669 \\
\hline & 20 & 50 & 500 & 50 & $27.08 \%$ & 1.52512 & $28.86 \%$ & 4.92925 \\
\hline & 50 & 50 & 50 & 20 & $98.60 \%$ & 1.86506 & $97.60 \%$ & 13.43552 \\
\hline & 50 & 50 & 50 & 40 & $98.60 \%$ & 1.83424 & $97.95 \%$ & 13.10968 \\
\hline & 50 & 50 & 50 & 60 & $98.55 \%$ & 1.91241 & $98.25 \%$ & 13.49794 \\
\hline \multirow{25}{*}{$50 \times 60$} & 0 & 0 & 0 & 0 & $4.75 \%$ & 1.26388 & $4.55 \%$ & 2.52770 \\
\hline & 10 & 0 & 0 & 0 & $11.35 \%$ & 1.48925 & $11.40 \%$ & 3.70839 \\
\hline & 20 & 0 & 0 & 0 & $33.65 \%$ & 1.75109 & $33.40 \%$ & 5.82381 \\
\hline & 30 & 0 & 0 & 0 & $65.70 \%$ & 1.82802 & $64.90 \%$ & 8.31756 \\
\hline & 0 & 50 & 0 & 0 & $4.70 \%$ & 1.26907 & $4.95 \%$ & 2.60854 \\
\hline & 0 & 200 & 0 & 0 & $4.75 \%$ & 1.23471 & $5.90 \%$ & 2.53725 \\
\hline & 0 & 300 & 0 & 0 & $5.35 \%$ & 1.19808 & $7.35 \%$ & 2.62291 \\
\hline & 0 & 0 & 50 & 0 & 0.0485 & 1.27867 & $5.15 \%$ & 2.57206 \\
\hline & 0 & 0 & 100 & 0 & $4.35 \%$ & 1.23893 & $4.45 \%$ & 2.40576 \\
\hline & 0 & 0 & 200 & 0 & $5.80 \%$ & 1.27524 & $5.50 \%$ & 2.62313 \\
\hline & 0 & 0 & 500 & 0 & $8.45 \%$ & 1.28708 & $6.50 \%$ & 2.64556 \\
\hline & 0 & 0 & 0 & 50 & $5.00 \%$ & 1.28525 & $4.85 \%$ & 2.71581 \\
\hline & 0 & 0 & 0 & 100 & $4.50 \%$ & 1.25700 & $4.70 \%$ & 2.57227 \\
\hline & 0 & 0 & 0 & 500 & $4.70 \%$ & 1.25102 & $5.10 \%$ & 2.51076 \\
\hline & 20 & 50 & 0 & 0 & $32.10 \%$ & 1.70264 & $32.30 \%$ & 5.72887 \\
\hline & 20 & 100 & 0 & 0 & $33.55 \%$ & 1.74878 & $32.50 \%$ & 5.76105 \\
\hline & 20 & 200 & 0 & 0 & $33.30 \%$ & 1.72065 & $34.35 \%$ & 5.71531 \\
\hline & 20 & 500 & 0 & 0 & $33.90 \%$ & 1.61848 & $46.30 \%$ & 5.77714 \\
\hline & 30 & 50 & 100 & 0 & $62.55 \%$ & 1.78912 & $61.80 \%$ & 8.08037 \\
\hline & 30 & 50 & 200 & 0 & $60.50 \%$ & 1.79929 & $58.60 \%$ & 7.76765 \\
\hline & 30 & 50 & 500 & 0 & $56.35 \%$ & 1.78203 & $54.00 \%$ & 7.76498 \\
\hline & 20 & 20 & 20 & 20 & $32.55 \%$ & 1.70930 & $33.05 \%$ & 5.60510 \\
\hline & 30 & 50 & 50 & 50 & $63.10 \%$ & 1.86549 & $61.65 \%$ & 8.63149 \\
\hline & 50 & 50 & 50 & 50 & $97.75 \%$ & 1.88146 & $97.10 \%$ & 13.27029 \\
\hline & 50 & 100 & 50 & 50 & $97.60 \%$ & 1.89668 & $96.90 \%$ & 13.30456 \\
\hline
\end{tabular}



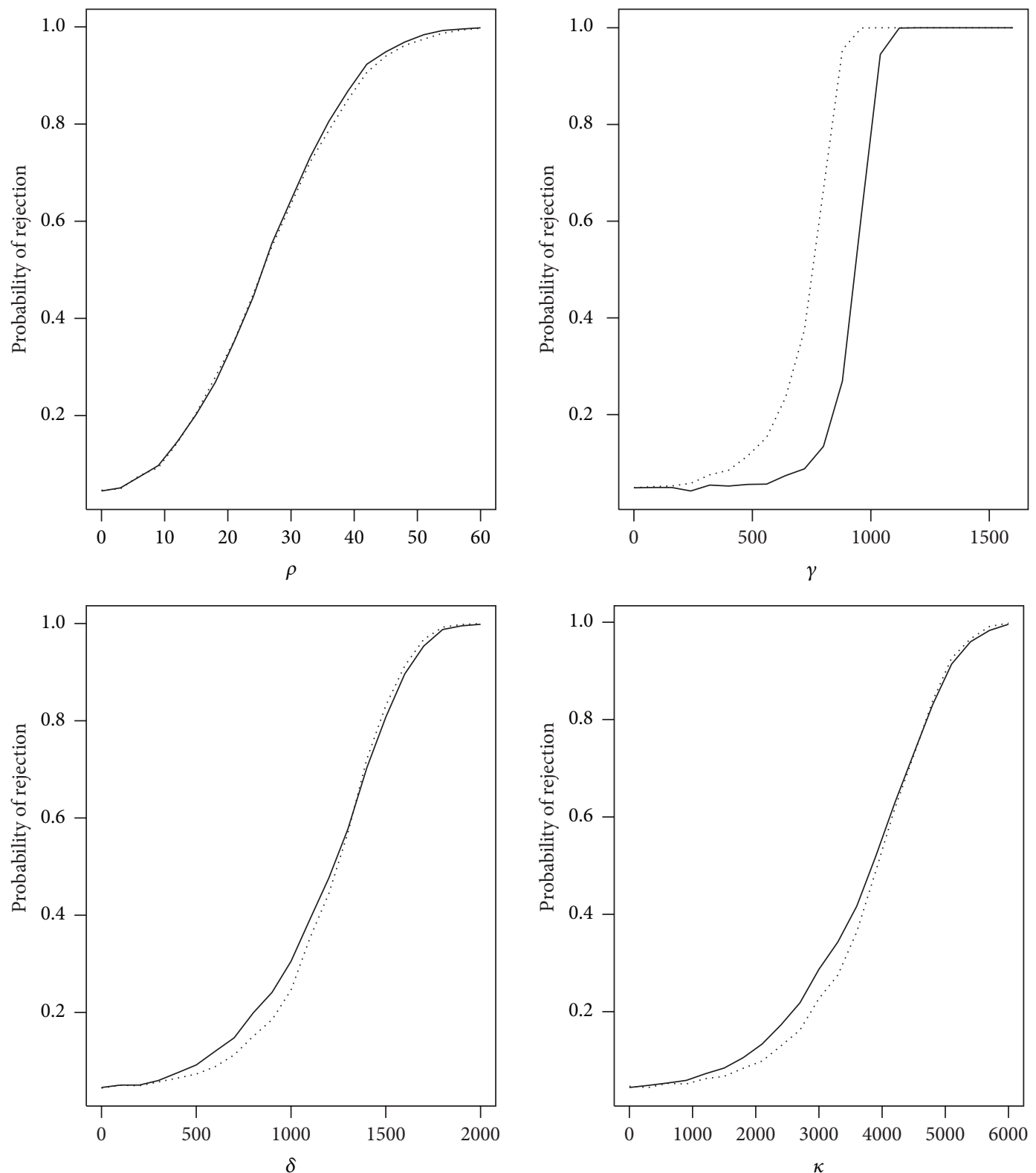

FIgURE 1: The empirical power functions of the KS test (smooth line) and CvM test (dotted line) for the 4-variate model using $50 \times 60$-point regular lattice. The simulation is under 10000 runs.

6.0742 and 7.9910 which are approximated by simulation. For $\rho=\gamma=\delta=\kappa=0$ the values of $\widehat{\alpha}_{\mathrm{KS}}$ and $\widehat{\alpha}_{\mathrm{CvM}}$ fluctuate around 0.05 as it should be. This means that, independent of the selected number of the lattice points, both tests attain the specified level of significance.

Furthermore, Figure 1 exhibits the graphs of the empirical power function of the KS and CvM tests for $\alpha=0.05$ associated with hypothesis $H_{0}$ specified above against $H_{1}$ : $g_{i} \notin \mathbf{W}_{i}$, for $i=1,2,3,4$. For the four cases we generate the error vectors independently from the same 4-variate normal distribution mentioned above. In the clockwise direction the left-top panel presents the graphs of the power function for testing $H_{0}$ against $H_{1}: g_{1} \notin \mathbf{W}_{1}$, the right-top panel is for $H_{0}$ against $H_{1}: g_{2} \notin \mathbf{W}_{2}$, the right-bottom is for $H_{0}$ against
$H_{1}: g_{3} \notin \mathbf{W}_{3}$, and left-bottom is for $H_{0}$ against $H_{1}: g_{4} \notin \mathbf{W}_{4}$. The common characteristic of the tests is that the power gets larger as the the model moves away from $H_{0}$. The KS tests represented by smooth line tend to have slightly larger power. However, somewhat unexpectedly, in the second case, the CvM test has much larger power.

\section{Example of Application}

In this example, the proposed method is applied to a mining data studied in Tahir [20]. As introduced in Section 1, the data consist of a simultaneous measurement of the percentages of Nickel (Ni), Cobalt (Co), Ferum (Fe), and other substances like Calcium-Monoxide $(\mathrm{CaO})$, Silicon-Dioxide $\left(\mathrm{SiO}_{2}\right)$, and 
TABLE 2: Pearson's correlation matrix of the percentages of $\mathrm{CaO}, \log \mathrm{SiO}_{2}, \log \mathrm{MgO}, \mathrm{Ni}, \log \mathrm{Fe}$, and Co observed over a regular lattice of size $7 \times 14$. Source of data: Tahir [20].

\begin{tabular}{|c|c|c|c|c|c|c|}
\hline & $\mathrm{CaO}$ & $\log \mathrm{SiO}_{2}$ & $\log \mathrm{MgO}$ & $\mathrm{Ni}$ & $\log \mathrm{Fe}$ & $\mathrm{Co}$ \\
\hline $\mathrm{CaO}$ & 1.0000 & 0.3949 & 0.4045 & -0.1285 & -0.1167 & -0.0665 \\
\hline $\log \mathrm{SiO}_{2}$ & 0.3949 & 1.0000 & 0.8459 & -0.0003 & -0.5414 & -0.4556 \\
\hline $\log \mathrm{MgO}$ & 0.4045 & 0.8459 & 1.0000 & -0.1331 & -0.4968 & -0.3134 \\
\hline $\mathrm{Ni}$ & -0.1285 & -0.0003 & -0.1331 & 1.0000 & 0.1652 & 0.1068 \\
\hline $\log \mathrm{Fe}$ & -0.1166 & -0.5414 & -0.4968 & 0.1652 & 1.0000 & 0.5937 \\
\hline $\mathrm{Co}$ & -0.0665 & -0.4556 & -0.3134 & 0.1068 & 0.5937 & 1.0000 \\
\hline
\end{tabular}

Magnesium-Monoxide ( $\mathrm{MgO})$. The sample was obtained by drilling bores set according to a three-dimensional lattice of size $7 \times 14 \times 10$ with 7 equidistance rows running west to east, 14 equidistance columns running south to north, and 10 equidistance depths from the surface of the earth to the bottom. To simplify the computation of the test statistics we consider the experimental design as a two-dimensional lattice of size $7 \times 14$ by taking the average value of the samples measured in the same position. We further assume that the exploration region is given by a closed rectangle so that by suitable rescaling it can be transformed into a closed unit rectangle $\mathbf{I}^{2}$. Table 2 exhibits, respectively, the pairs scatter plot and Pearson's correlation coefficient among the percentages of $\mathrm{Ni}, \mathrm{CaO}, \mathrm{Co}$, the logarithm of the percentages of $\mathrm{SiO}_{2}\left(\log \mathrm{SiO}_{2}\right), \mathrm{MgO}(\log \mathrm{MgO})$, and $\mathrm{Fe}(\log \mathrm{Fe})$. By this reason a multivariate analysis must be conducted in the statistical modelling taking into account the unknown covariance matrix of the vector of the variables. Furthermore, based on the individual scatter plot of the samples which are not presented in this work, it can be inferred that polynomials of lower order seem to be adequate to approximate the population model. More precisely, let $\mathbf{Y}:=\left(Y_{1}, Y_{2}, Y_{3}, Y_{4}, Y_{5}, Y_{6}\right)^{\top}$ be the vector of observations representing the observed percentages of $\mathrm{CaO}, \log \mathrm{SiO}_{2}$, $\log \mathrm{MgO}, \mathrm{Co}, \mathrm{Ni}$, and $\log \mathrm{Fe}$, respectively. We aim to test the hypothesis

$$
\begin{aligned}
& H_{0}: \mathbf{E}(Y) \\
& =\left(\begin{array}{c}
\beta_{11} \\
\beta_{21}+\beta_{22} t+\beta_{23} s \\
\beta_{31}+\beta_{32} t+\beta_{33} s \\
\beta_{41}+\beta_{42} t+\beta_{43} s \\
\beta_{51}+\beta_{52} t+\beta_{53} s+\beta_{54} t^{2}+\beta_{55} t s+\beta_{56} s^{2} \\
\beta_{61}+\beta_{62} t+\beta_{63} s+\beta_{64} t^{2}+\beta_{65} t s+\beta_{66} s^{2}
\end{array}\right), \\
& 0 \leq t, s \leq 1,
\end{aligned}
$$

for some unknown constants $\beta_{i j}, i=1,2,3,4,5,6$ and $j=$ $1, \ldots, d_{i}$, with $d_{1}=1, d_{2}=d_{3}=d_{4}=3$ and $d_{5}=d_{6}=6$. For this case we have $\mathbf{W}_{1}:=\left[f_{1}\right], \mathbf{W}_{2}=\mathbf{W}_{3}=\mathbf{W}_{4}:=\left[f_{1}, f_{2}, f_{3}\right]$, and $\mathbf{W}_{5}=\mathbf{W}_{6}:=\left[f_{1}, f_{2}, f_{3}, f_{4}, f_{5}, f_{6}\right]$, with $f_{1}(t, s):=1$, $f_{2}(t, s)=t, f_{3}(t, s)=s, f_{4}(t, s)=t^{2}, f_{5}(t, s)=t s$, and $f_{6}(t, s)=s^{2}$.
We obtained the values $\mathrm{KS}_{7 \times 14 ; \mathscr{A}}=1.44109$ and $\mathrm{CvM}_{7 \times 14 ; \mathscr{A}}=0.936687$ with the associated simulated $p$ values of 0.98350 and 0.93670 , respectively. We notice that in the computation we consider the $\operatorname{VCC}\{[0, t] \times[0, s]$ : $0 \leq t, s \leq 1\}$ as the index sets instead of $\mathscr{A}$. Hence when using the KS test as well as CvM test the hypothesis will be not rejected for almost all commonly used values of $\alpha$. There exists a significant evidence that the assumed model is appropriate for describing the functional relationship between the experimental conditional and the percentages of those elements.

In the practice some computational difficulties appear for testing using our proposed method. First, to the knowledge of the authors, the analytical formula for computing the critical and $p$ values of the tests have been not yet available in the literatures; therefore we need to approximate them by simulation using computer. Second, although the test procedures are established for a much larger family of sets $\mathscr{A}$, in the application the computation is always restricted to the VCC of subsets of $\mathbf{I}^{d}$ like that of $\left\{\prod_{i=1}^{d}\left[0, t_{i}\right] \subset \mathbf{I}^{d}: 0<t_{i} \leq\right.$ $1, i=1, \ldots, d\}$ or $\left\{\prod_{i=1}^{d}\left[s_{i}, t_{i}\right] \subset \mathbf{I}^{d}: 0 \leq s_{i}<t_{i} \leq 1, i=\right.$ $1, \ldots, d\}$.

\section{Concluding Remark}

In this article we have developed an asymptotic method for checking the validity of a general multivariate spatial regression model by considering the multidimensional setindexed partial sums of the residuals. For the calibration of the distribution of the test statistics we propose the residual based bootstrap for multivariate regression. It is shown by applying imitation technique that the residual bootstrap resampling technique is consistent. In a simulation study the finite sample size behavior of the KS and CvM statistics is investigated in greater detail. For the first-order model CvM test has much larger power, whereas for constant, secondorder, and third-order models the powers of the two tests are almost the same.

Other possibilities of tests for multidimensional case can be obtained by incorporating a sampling technique according to an arbitrary experimental design. Sometimes because of technical, economic, or ecological reason, practitioners will not or cannot sample the observations equidistantly. One possible approach is to sample according to a continuous probability measure; see, for example, the sampling method 
proposed in Bischoff [11]. Under this concern we get the socalled weighted KS and CvM tests which can be viewed as generalization of the KS and CvM tests studied in this paper.

Instead of considering the least squares residuals of the observations we can also define a test by directly investigating the partial sums of the observations. The limit process will be given by a type of signal plus a noise which is given by the multidimensional set-indexed Brownian sheet. Observing the limit process we can formulate likelihood ratio test based on the Cameron-Matrin-Girsanov density formula of the limit process. Establishing such type of test will be of our concern in our future research project.

\section{Appendix}

\section{A. Function of Bounded Variation on $\mathbf{I}^{d}$}

Definition A.1. Let $f: \mathbb{R}^{d} \rightarrow \mathbb{R}$ be a real-valued function with $d$ variables. For $\alpha_{k}, \beta_{k} \in \mathbb{R}$, let $\Delta_{\alpha_{k}}^{\beta_{k}} f$ be a real-value function defined on $\mathbb{R}^{d}$, given by

$$
\begin{aligned}
\Delta_{\alpha_{k}}^{\beta_{k}} f:= & f\left(x_{1}, \ldots, x_{k-1}, \beta_{k}, x_{k+1}, \ldots, x_{d}\right) \\
& -f\left(x_{1}, \ldots, x_{k-1}, \alpha_{k}, x_{k+1}, \ldots, x_{d}\right),
\end{aligned}
$$

for $k=1, \ldots, d$. Furthermore, for $\alpha:=\left(\alpha_{k}\right)_{k=1}^{d}$ and $\beta:=$ $\left(\beta_{k}\right)_{k=1}^{d} \in \mathbb{R}^{d}, \Delta_{\alpha}^{\beta} f$ is defined on $\mathbb{R}^{d}$ recursively starting from the last components of $\alpha$ and $\beta$. More precisely,

$$
\Delta_{\alpha}^{\beta} f:=\Delta_{\alpha_{1}}^{\beta_{1}}\left(\cdots\left(\Delta_{\alpha_{d-1}}^{\beta_{d-1}}\left(\Delta_{\alpha_{d}}^{\beta_{d}} f\right)\right) \cdots\right) .
$$

Let $\left\{j_{1}, \ldots, j_{d}\right\}$ be permutation of $\{1,2, \ldots, d\}$; then it holds that

$$
\begin{aligned}
\Delta_{\alpha}^{\beta} f & =\Delta_{\alpha_{j_{1}}}^{\beta_{j_{1}}}\left(\cdots\left(\Delta_{\alpha_{j_{d-1}}}^{\beta_{j_{d-1}}}\left(\Delta_{\alpha_{j_{d}}}^{\beta_{j_{d}}} f\right)\right) \cdots\right) \\
& =\Delta_{\alpha_{1}}^{\beta_{1}}\left(\cdots\left(\Delta_{\alpha_{d-1}}^{\beta_{d-1}}\left(\Delta_{\alpha_{d}}^{\beta_{d}} f\right)\right) \cdots\right) .
\end{aligned}
$$

This means that the operation of $\Delta_{\alpha}^{\beta} f$ does not depend on the order. By this reason we write $\Delta_{\alpha}^{\beta} f$ by $\Delta_{\alpha_{1}}^{\beta_{1}} \cdots \Delta_{\alpha_{d-1}}^{\beta_{d-1}} \Delta_{\alpha_{d}}^{\beta_{d}} f$ ignoring the brackets. The reader is referred to Yeh [33] and Elstrodt [34], pp. 44-45.

Definition A.2 (see Yeh [33]). Let $\Gamma_{k}:=\left\{\left[x_{k_{0}}, x_{k_{1}}\right]\right.$, $\left.\left[x_{k_{1}}, x_{k_{2}}\right], \ldots,\left[x_{k_{M_{k}-1}}, x_{k_{M_{k}}}\right]\right\}$ be a collection of $M_{k}$ rectangles on the unit interval $[0,1]$ with $0=x_{k_{0}} \leq x_{k_{1}} \leq \cdots \leq x_{k_{M_{k}}}=$ 1 , for $k=1, \ldots, d$. The Cartesian product $\mathscr{K}:=\prod_{k=1}^{d} \Gamma_{k}$ which consists of $M_{1} \times M_{2} \times \cdots \times M_{d}$ rectangles is called a nonoverlapping finite exact cover of $\mathbf{I}^{d}$. The family of all nonoverlapping finite exact cover of $\mathbf{I}^{d}$ is denoted by $\mathscr{J}(\mathscr{K})$.

Definition A.3 (see Yeh [33]). For $1 \leq w_{k} \leq M_{k}$, with $k=$ $1, \ldots, d$, let $\mathbf{J}_{w_{1} \cdots w_{d}}$ be the element of $\mathscr{K}$ defined by $\mathbf{J}_{w_{1} \cdots w_{d}}:=$ $\prod_{k=1}^{d}\left[x_{k_{w_{k}-1}}, x_{k_{w_{k}}}\right]$. Let $\psi: \mathbf{I}^{d} \rightarrow \mathbb{R}$ be a real-valued function on $\mathbf{I}^{d}$. Operator $\Delta_{\mathbf{J}_{w_{1} \cdots w_{d}}}$ acting on a function $\psi$ is defined by

$$
\Delta_{\mathbf{J}_{w_{1} \cdots w_{d}}} \psi:=\Delta_{x_{1_{w_{1}-1}}}^{x_{1_{w_{1}}}} \Delta_{x_{2_{w_{2}-1}}}^{x_{2_{w_{2}}}} \cdots \Delta_{x_{d_{w_{d}-1}}}^{x_{d_{w_{d}}}} \psi
$$

The variation of $\psi$ over the finite exact cover $\mathscr{K}$ is defined by

$$
v(\psi ; \mathscr{K}):=\sum_{w_{1}=1}^{M_{1}} \ldots \sum_{w_{d}=1}^{M_{d}}\left|\Delta_{\mathbf{J}_{w_{1} \cdots w_{d}}} \psi\right|
$$

Accordingly, the total variation of $\psi$ over $\mathbf{I}^{d}$ is defined by

$$
V\left(\psi ; \mathbf{I}^{d}\right):=\sup _{\mathscr{K} \in \mathscr{I}(\mathscr{K})} v(\psi ; \mathscr{K})
$$

Furthermore, function $\psi$ is said to have bounded variation in the sense of Vitaly on $\mathbf{I}^{d}$ if there exists a real number $M>0$ such that $V\left(\psi ; \mathbf{I}^{d}\right) \leq M$ for some real number $M>0$. The class of such functions is denoted by $\operatorname{BVV}\left(\mathbf{I}^{d}\right)$.

Definition A.4 (see Yeh [33]). Let $\left(x_{k}\right)_{k=1}^{d}$ be a variable in $\mathbf{I}^{d}$. For fixed $k$, let $\mathbf{I}^{k}:=[0,1]^{k}$ be a $k$-dimensional unit closed rectangle constructed in the following way. We choose $d-$ $k$ components of the variable $\left(x_{k}\right)_{k=1}^{d}$. For each choice from all possible elements of the set $C_{d-k}^{d}$, we set each $x_{i}$ with 0 or 1 and let the remaining $k$ variables satisfy $0 \leq x_{i} \leq 1$. Then for each $k$ we get $2^{d-k}\left|C_{d-k}^{d}\right|$ unit closed rectangles $\mathbf{I}^{k}$. For convention we denote the collection of all $2^{d-k}\left|C_{d-k}^{d}\right|$ of closed rectangles $\mathbf{I}^{k}$ by $\mathscr{B}^{k}$ and the $j$ th element of $\mathscr{B}^{k}$ will be denoted by $\mathbf{I}_{j}^{k}$. Function $\psi$ is said to have bounded variation in the sense of Hardy on $\mathbf{I}^{d}$, if and only if for each $k=1, \ldots, d$ and $j=1, \ldots, 2^{d-k}\left|C_{d-k}^{d}\right|$ there exists a real number $M_{j k}>0$ such that $V\left(\psi_{\mathbf{I}_{j}^{k}}(\cdot) ; \mathbf{I}_{j}^{k}\right) \leq M_{j k}$, where, for $k=1, \ldots, d$ and $j=$ $1, \ldots, 2^{d-k}\left|C_{d-k}^{d}\right|, \psi_{\mathbf{I}_{j}^{k}}(\cdot)$ is a function with $k$ variables obtained from the function $\psi\left(x_{1}, x_{2} \ldots, x_{d}\right)$ by setting the $d-k$ selected variables with 0 or 1 , whereas the remaining $k$ variables lie in the interval $[0,1]$. The class of such functions will be denoted by $\mathrm{BV}_{H}\left(\mathbf{I}^{d}\right)$.

\section{B. Integration by Parts on $\mathbf{I}^{d}$}

For family $\mathscr{B}^{k}$ defined in Definition A.4, let $\mathscr{I}:=\bigcup_{k=0}^{d} \mathscr{B}^{k}$, where, for $k=0$, the family $\mathscr{B}^{0}$ is a collection of $2^{d}$ different points in $\mathbf{I}^{d}$. As an example, for $d=2$, we have $\mathscr{B}^{0}=\left\{\mathbf{I}_{1}^{0}=\right.$ $\left.(0,0), \mathbf{I}_{2}^{0}=(0,1), \mathbf{I}_{3}^{0}=(1,0), \mathbf{I}_{4}^{0}=(1,1)\right\}$. For each $k=1, \ldots, d$ and $j=1, \ldots, 2^{d-k}\left|C_{k-d}^{d}\right|$, let $\sharp\left(\mathbf{I}_{j}^{k}\right)$ be the number of 1 's appearing in $\mathbf{I}_{j}^{k}$. Next, let $\varphi$ and $\psi$ be defined on $\mathbf{I}^{d}$. If $\varphi$ is Riemann-Stieltjes integrable with respect to $\psi$ on $\mathbf{I}_{j}^{k} \in \mathscr{B}^{k}$, we denote the integral by $\int_{\mathbf{I}_{j}^{k}}^{(R)} \varphi d^{k} \psi$. For $k=0$, it is understood that $\int_{\mathbf{I}_{j}^{0}}^{(R)} \varphi d^{0} \psi$ is defined as the product of $\varphi$ and $\psi$ at that point of $\mathbf{I}_{j}^{0}$ (see Yeh [33]).

Theorem B.1 (integration by parts (see Yeh [33])). Let $\varphi$ be Riemann-Stieltjes integrable with respect to $\psi$ on each member 
of $\mathscr{I}$. Then $\psi$ is Riemann-Stieltjes integrable with respect to $\varphi$ on $\mathbf{I}^{d}$, and we have the formula

$$
\begin{aligned}
& \int_{\mathbf{I}^{d}}^{(R)} \psi\left(x_{1}, \ldots, x_{d}\right) d \varphi\left(x_{1}, \ldots, x_{d}\right) \\
& =\sum_{k=0}^{d} \sum_{j=1}^{2^{d-k}\left|C_{d-k}^{d}\right|}(-1)^{\left(d-\sharp\left(\mathbf{I}_{j}^{k}\right)\right)} \int_{\mathbf{I}_{j}^{k}}^{(R)} \varphi d^{k} \psi .
\end{aligned}
$$

Moreover, if $\psi$ have bounded variation in the sense of Hardy on $\mathbf{I}^{d}$ and $\varphi$ is continuous on $\mathbf{I}^{d}$, then we have the inequality

$$
\begin{aligned}
& \left|\int_{\mathbf{I}^{d}}^{(R)} \psi\left(x_{1}, \ldots, x_{d}\right) d \varphi\left(x_{1}, \ldots, x_{d}\right)\right| \\
& \quad \leq\|\varphi\|_{\infty}\left(2^{d}\|\psi\|_{\infty}+\sum_{k=1}^{d} \sum_{j=1}^{2^{d-k}\left|C_{d-k}^{d}\right|} V\left(\psi_{\mathbf{I}_{j}^{k}}(\cdot) ; \mathbf{I}_{j}^{k}\right)\right) .
\end{aligned}
$$

\section{Some Property of the Partial Sums Operator}

Lemma C.1 (see Bischoff and Somayasa [9]). For every onedimensional pyramidal array $A_{n_{1} \cdots n_{d}}:=\left(a_{j_{1} \cdots j_{d}}\right)_{j_{1}=1, \ldots, j_{d}=1}^{n_{1}, \ldots, n_{d}}$, it holds that $\mathbf{V}_{n_{1} \cdots n_{d}}^{(i)}\left(A_{n_{1} \cdots n_{d}}\right) \in \mathscr{H}_{B}$, where $\mathscr{H}_{B}$ is the subspace defined in (15). Furthermore, for any arrays $A_{n_{1} \cdots n_{d}}:=$ $\left(a_{j_{1} \cdots j_{d}}\right)_{j_{1}=1, \ldots, j_{d}=1}^{n_{1}, \ldots, n_{d}}$ and $B_{n_{1} \cdots n_{d}}:=\left(b_{j_{1} \cdots j_{d}}\right)_{j_{1}=1, \ldots, j_{d}=1}^{n_{1}, \ldots, n_{d}}$, we have

$$
\begin{aligned}
& \left\langle V_{n_{1} \cdots n_{d}}^{(i)}\left(A_{n_{1} \cdots n_{d}}\right), V_{n_{1} \cdots n_{d}}^{(i)}\left(B_{n_{1} \cdots n_{d}}\right)\right\rangle_{\mathscr{H}_{B}} \\
& =\left\langle A_{n_{1} \cdots n_{d}}, B_{n_{1} \cdots n_{d}}\right\rangle_{\mathbb{R}^{n_{1} \times \cdots \times n_{d}}},
\end{aligned}
$$

where $V_{n_{1} \cdots n_{d}}^{(i)}$ is the one-dimensional component of the partial sums operator $\mathbf{V}_{n_{1} \cdots n_{d}}$.

Proof. Associated with $A_{n_{1} \cdots n_{d}}$ we can construct a step function $s_{A_{n_{1} \cdots n_{d}}}: \mathbf{I}^{d} \rightarrow \mathbb{R}$ defined by

$$
s_{A_{n_{1} \cdots n_{d}}}(\mathbf{t}):=\sum_{j_{1}=1}^{n_{1}} \cdots \sum_{j_{d}=1}^{n_{d}} a_{j_{1} \cdots j_{d}} \mathbf{1}_{C_{j_{1} \cdots j_{d}}}(\mathbf{t}), \quad \mathbf{t} \in \mathbf{I}^{d}
$$

where $C_{j_{1} \cdots j_{d}}=\prod_{k=1}^{d}\left(\left(j_{k}-1\right) / n_{k}, j_{k} / n_{k}\right]$, for $1 \leq j_{k} \leq n_{k}$. For any $B \in \mathscr{A}$, it holds that

$$
\begin{aligned}
h_{s_{A_{n_{1} \cdots n_{d}}}}(B) & :=\int_{B} s_{A_{n_{1} \cdots n_{d}}}(\mathbf{t}) \lambda_{\mathbf{I}}^{d}(d \mathbf{t}) \\
& =\frac{1}{\sqrt{n_{1} \cdots n_{d}}} V_{n_{1} \cdots n_{d}}^{(i)}\left(A_{n_{1} \cdots n_{d}}\right)(B) .
\end{aligned}
$$

Hence, $V_{n_{1} \cdots n_{d}}^{(i)}\left(A_{n_{1} \cdots n_{d}}\right) \in \mathscr{H}_{B}$ having $\sqrt{n_{1} \cdots n_{d}} s_{A_{n_{1} \cdots n_{d}}}$ as the $L_{2}\left(\lambda_{\mathrm{I}}^{d}\right)$ density. By the definition of the inner product $\langle\cdot, \cdot\rangle_{\mathscr{H}_{B}}$, we further get

$$
\begin{aligned}
& \left\langle V_{n_{1} \cdots n_{d}}^{(i)}\left(A_{n_{1} \cdots n_{d}}\right), V_{n_{1} \cdots n_{d}}^{(i)}\left(B_{n_{1} \cdots n_{d}}\right)\right\rangle_{\mathscr{H}_{B}} \\
& =\int_{\mathbf{I}^{d}} \sqrt{n_{1} \cdots n_{d}} s_{A_{n_{1} \cdots n_{d}}}(\mathbf{t}) \sqrt{n_{1} \cdots n_{d}} s_{B_{n_{1} \cdots n_{d}}}(\mathbf{t}) \\
& \cdot \lambda_{\mathbf{I}}^{d}(d \mathbf{t})=\sum_{j_{1}=1}^{n_{1}} \cdots \sum_{j_{d}=1}^{n_{d}} a_{j_{1} \cdots j_{d}} b_{j_{1} \cdots j_{d}} \\
& =\left\langle A_{n_{1} \cdots n_{d}}, B_{n_{1} \cdots n_{d}}\right\rangle_{\mathbb{R}^{n_{1} \times \cdots \times n_{d}}} .
\end{aligned}
$$

Lemma C.2 (see Bischoff and Somayasa [9]). For any $A_{n_{1} \cdots n_{d}}:=\left(a_{j_{1} \cdots j_{d}}\right)_{j_{1}=1, \ldots, j_{d}=1}^{n_{1}, \ldots, n_{d}}$ in $\mathbb{R}^{n_{1} \times \cdots \times n_{d}}$ it holds that, for $i=$ $1, \ldots, p$,

$$
\begin{aligned}
& V_{n_{1} \cdots n_{d}}^{(i)}\left(\operatorname{pr}_{\mathbf{W}_{i, n_{1} \cdots n_{d}}} A_{n_{1} \cdots n_{d}}\right) \\
& =p r_{\mathbf{W}_{i, n_{1} \cdots n_{d} \mathscr{H}}} V_{n_{1} \cdots n_{d}}^{(i)}\left(A_{n_{1} \cdots n_{d}}\right),
\end{aligned}
$$

where

$$
\begin{aligned}
\mathbf{W}_{i, n_{1} \cdots n_{d} \mathscr{H}_{B}} & :=\left\{V_{n_{1} \cdots n_{d}}^{(i)}\left(B_{n_{1} \cdots n_{d}}\right) \mid B_{n_{1} \cdots n_{d}} \in \mathbf{W}_{i, n_{1} \cdots n_{d}}\right\} \\
& \subset \mathscr{H}_{B} .
\end{aligned}
$$

Furthermore, by the definition of the component-wise projection, we finally get

$$
\begin{aligned}
& \mathbf{V}_{n_{1} \cdots n_{d}}\left(p r_{\prod_{i=1}^{p} \mathbf{w}_{i, n_{1} \cdots n_{d}}} \mathbf{A}_{n_{1} \cdots n_{d}}\right) \\
& =\left(p r_{\mathbf{W}_{i, n_{1} \cdots n_{d} \mathscr{C}_{B}}} V_{n_{1} \cdots n_{d}}^{(i)}\left(A_{n_{1} \cdots n_{d}}\right)\right)_{i=1}^{p},
\end{aligned}
$$

for every $p$-dimensional array $\mathbf{A}_{n_{1} \cdots n_{d}} \in \prod_{i=1}^{p} \mathbb{R}^{n_{1} \times \cdots \times n_{d}}$.

Proof. For fixed $i$, let $\left\{f_{i 1}\left(\Xi_{n_{1} \cdots n_{d}}\right), \ldots, f_{i d_{i}}\left(\Xi_{n_{1} \cdots n_{d}}\right)\right\}$ be an ONB of $\mathbf{W}_{i, n_{1} \cdots n_{d}}$. Then by Lemma C.1 the corresponding ONB of $\mathbf{W}_{i, n_{1} \cdots n_{d \mathscr{H}_{B}}}$ is given by the set

$$
\left\{\mathbf{V}_{n_{1} \cdots n_{d}}^{(i)}\left(f_{i 1}\left(\Xi_{n_{1} \cdots n_{d}}\right)\right), \ldots, \mathbf{V}_{n_{1} \cdots n_{d}}^{(i)}\left(f_{i d_{i}}\left(\Xi_{n_{1} \cdots n_{d}}\right)\right)\right\} .
$$

Hence, by the linearity of $\mathbf{V}_{n_{1} \cdots n_{d}}^{(i)}$ and by Lemma C.1, we get

$$
\begin{aligned}
& V_{n_{1} \cdots n_{d}}^{(i)}\left(\operatorname{pr}_{\mathbf{W}_{i, n_{1} \cdots n_{d}}} A_{n_{1} \cdots n_{d}}\right) \\
& =V_{n_{1} \cdots n_{d}}^{(i)}\left(\sum_{j=1}^{d_{i}}\left\langle f_{i j}\left(\Xi_{n_{1} \cdots n_{d}}\right), A_{n_{1} \cdots n_{d}}\right\rangle_{\mathbb{R}^{n_{1} \times \cdots \times n_{d}}}\right. \\
& \left.\quad \cdot f_{i j}\left(\Xi_{n_{1} \cdots n_{d}}\right)\right)
\end{aligned}
$$




$$
\begin{aligned}
& =\sum_{j=1}^{d_{i}}\left\langle f_{i j}\left(\Xi_{n_{1} \cdots n_{d}}\right), A_{n_{1} \cdots n_{d}}\right\rangle_{\mathbb{R}^{n_{1} \times \cdots \times n_{d}}} \\
& \cdot V_{n_{1} \cdots n_{d}}^{(i)}\left(f_{i j}\left(\Xi_{n_{1} \cdots n_{d}}\right)\right) \\
& =\sum_{j=1}^{d_{i}}\left\langle V_{n_{1} \cdots n_{d}}^{(i)}\left(f_{i j}\left(\Xi_{n_{1} \cdots n_{d}}\right)\right), V_{n_{1} \cdots n_{d}}^{(i)}\left(A_{n_{1} \cdots n_{d}}\right)\right\rangle_{\mathscr{H}_{B}} \\
& \cdot V_{n_{1} \cdots n_{d}}^{(i)}\left(f_{i j}\left(\Xi_{n_{1} \cdots n_{d}}\right)\right) \\
& =\operatorname{pr}_{\mathbf{W}_{i, n_{1} \cdots n_{d} \mathscr{C}_{B}}} V_{n_{1} \cdots n_{d}}^{(i)}\left(A_{n_{1} \cdots n_{d}}\right) .
\end{aligned}
$$

Lemma C.3 (see Bischoff and Somayasa [9]). Let $\left\{\widetilde{s}_{i 1}^{\left(n_{1} \cdots n_{d}\right)}, \ldots\right.$, $\left.\widetilde{s}_{i d_{i}}^{\left(n_{1} \cdots n_{d}\right)}\right\}$ be an orthonormal set in $L_{2}\left(\lambda_{\mathrm{I}}^{d}\right)$ obtained by the Gram-Schmidt procedure from the step functions:

$$
\begin{aligned}
& s_{i j}^{\left(n_{1} \cdots n_{d}\right)}(\mathbf{t}) \\
& :=\sum_{j_{1}=1}^{n_{1}} \cdots \sum_{j_{d}=1}^{n_{d}} f_{i j}\left(\frac{j_{1}}{n_{1}}, \ldots, \frac{j_{d}}{n_{d}}\right) \mathbf{1}_{C_{j_{1} \cdots j_{d}}}(\mathbf{t}), \\
& \quad \mathbf{t} \in \mathbf{I}^{d},
\end{aligned}
$$

for $i=1, \ldots, p$, and $j=1, \ldots, d_{i}$. Then $\left\{h_{\widetilde{s}_{i 1}\left(n_{1} \ldots n_{d}\right)}, \ldots, h_{\left.\widetilde{s}_{i d_{i}} \ldots n_{d}\right)}\right\}$ is an ONB of $\mathbf{W}_{i, n_{1} \cdots n_{d \mathscr{H}_{B}}}$. The projection of any function $u_{n} \in \mathscr{H}_{B}$ into $\mathbf{W}_{i, n_{1} \cdots n_{d \mathscr{H}_{B}}}$ with respect to this basis is given by

$$
\begin{aligned}
& p r_{\mathbf{W}_{i, n_{1} \cdots n_{d \mathscr{H}_{B}}}} u_{n}=\sum_{j=1}^{d_{i}}\left\langle h_{\widetilde{s}_{i j}\left(n_{1} \cdots n_{d}\right)}, u_{n}\right\rangle_{\mathscr{H}_{B}} h_{\widetilde{s}_{i j}\left(n_{1} \cdots n_{d}\right)} \\
& =\sum_{j=1}^{d_{i}} \int_{\mathrm{I}^{d}}^{(R)} \widetilde{s}_{i j}^{\left(n_{1} \cdots n_{d}\right)}(\mathbf{t}) d u(\mathbf{t}) h_{\left.\widetilde{s}_{\tilde{s}_{j}} \cdots n_{d}\right)} .
\end{aligned}
$$

Moreover, if, for $i=1, \ldots, p$ and $j=1, \ldots, d_{i}, f_{i j}$ is continuous on $\mathbf{I}^{d}$ and $\left\{f_{i 1}, \ldots, f_{i d_{i}}\right\}$ is an ONB of $\mathbf{W}_{i}$, then $\| \widetilde{s}_{i j}\left(n_{1} \cdots n_{d}\right)$ $f_{i j} \|_{\infty} \rightarrow 0$ as $n_{1}, \ldots, n_{d} \rightarrow \infty$. Consequently, it also holds that $\left\|h_{\widetilde{s}_{i j}^{\left(n_{1} \cdots n_{d}\right)}}-h_{f_{i j}}\right\|_{\mathscr{A}} \rightarrow 0$, for $n_{1}, \ldots, n_{d} \rightarrow \infty$.

Proof. Since $h_{s_{i j}^{\left(n_{1} \cdots n_{d}\right)}}=\left(1 / \sqrt{n_{1} \cdots n_{d}}\right) V_{n_{1} \cdots n_{d}}^{(i)}\left(f_{i j}\left(\Xi_{n_{1} \cdots n_{d}}\right)\right)$, by the linearity of $V_{n_{1} \cdots n_{d}}^{(i)}$ it follows that $\left\{h_{s_{i 1}\left(n_{1} \cdots n_{d}\right)}, \ldots, h_{s_{i d_{i}}\left(n_{1} \cdots n_{d}\right)}\right\}$ builds a basis for $\mathbf{W}_{i, n_{1} \cdots n_{d \mathscr{H}_{B}}}$ whenever the set $\left\{f_{i 1}\left(\Xi_{n_{1} \cdots n_{d}}\right), \ldots\right.$, $\left.f_{i d_{i}}\left(\Xi_{n_{1} \cdots n_{d}}\right)\right\}$ is a basis of $\mathbf{W}_{i, n_{1} \cdots n_{d}}$. Furthermore, if $f_{i j}$ is continuous on $\mathbf{I}^{d}$, it can be shown that $\left\|s_{i j}^{\left(n_{1} \cdots n_{d}\right)}-f_{i j}\right\|_{\infty} \rightarrow 0$ as $n_{1}, \ldots, n_{d} \rightarrow \infty$. Hence, by the definition of the GramSchmidt process and also by the continuity of $\|\cdot\|_{L_{2}\left(\lambda_{\mathrm{I}}^{d}\right)}$, we can further show that $\left\|h_{\widetilde{s}_{i j}\left(n_{1} \cdots n_{d}\right)}-h_{f_{i j}}\right\|_{\mathscr{A}} \rightarrow 0$ as $n_{1}, \ldots, n_{d} \rightarrow \infty$. The last assertion is immediately obtained from the definition of $h_{\widetilde{s}_{i j}\left(n_{1} \cdots n_{d}\right)}$ and $h_{f_{i j}}$.
Proposition C.4. The p-dimensional projection $\operatorname{pr}_{\prod_{i=1}^{p} \mathbf{w}_{i, n_{1} \cdots n_{d \mathscr{H}_{B}}}}$ is continuous uniformly on the space of continuous function $\mathscr{C}^{p}(\mathscr{A})$ for all $n_{1} \geq 1, \ldots, n_{d} \geq 1$.

Proof. Let $\mathbf{w}_{1}:=\left(w_{1 i}\right)_{i=1}^{p}$ and $\mathbf{w}_{2}:=\left(w_{2 i}\right)_{i=1}^{p}$ be any functions in $\mathscr{C}^{P}(\mathscr{A})$. Then, by the definition and the inequality presented in Theorem B.1, for any $A \in \mathscr{A}$ we have

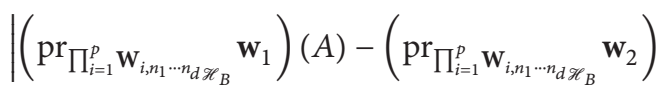

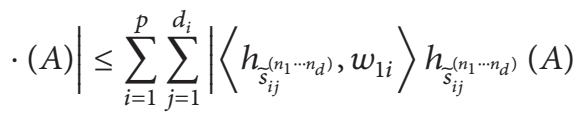

$$
\begin{aligned}
& -\left\langle h_{\widetilde{s}_{i j}\left(n_{1} \cdots n_{d}\right)}, w_{2 i}\right\rangle h_{\widetilde{s}_{i j}^{\left(n_{1} \cdots n_{d}\right)}}(A) \mid \\
& =\sum_{i=1}^{p} \sum_{j=1}^{d_{i}}\left|\left\langle h_{\widetilde{s}_{i j}^{\left(n_{1} \cdots n_{d}\right)}}, w_{1 i}-w_{2 i}\right\rangle\right|\left|h_{\widetilde{s}_{i j}^{\left(n_{1} \cdots n_{d}\right)}}(A)\right| \\
& \leq \sum_{i=1}^{p} \sum_{j=1}^{d_{i}}\left\|w_{1 i}-w_{2 i}\right\|_{\mathscr{A}}\left\|f_{i j}\right\|_{\infty}\left(2^{d}\left\|f_{i j}\right\|_{\infty}\right. \\
& \left.+\sum_{k=1}^{d} \sum_{\ell=1}^{2^{d-k}\left|C_{d-k}^{d}\right|} V\left(f_{i j_{\mathbf{I}_{\ell}^{k}}}(\cdot) ; \mathbf{I}_{\ell}^{k}\right)\right) \leq\left\|\mathbf{w}_{1}-\mathbf{w}_{2}\right\|_{\mathscr{A}} K,
\end{aligned}
$$

for some constant $K$ defined by

$$
\begin{aligned}
K: & =\sum_{i=1}^{p} \sum_{j=1}^{d_{i}}\left\|f_{i j}\right\|_{\infty} \\
& \cdot\left(2^{d}\left\|f_{i j}\right\|_{\infty}+\sum_{k=1}^{d} \sum_{\ell=1}^{2^{d-k}\left|C_{d-k}^{d}\right|} V\left(f_{i j_{\mathbf{I}_{\ell}^{k}}}(\cdot) ; \mathbf{I}_{\ell}^{k}\right)\right) .
\end{aligned}
$$

Hence we get

$$
\begin{aligned}
& \left\|\operatorname{pr}^{\prod_{i=1}^{p} \mathbf{w}_{i, n_{1} \cdots n_{d \mathscr{H}}}} \mathbf{w}_{1}-\operatorname{pr}_{\prod_{i=1}^{p} \mathbf{w}_{i, n_{1} \cdots n_{d \mathscr{H}}}} \mathbf{w}_{2}\right\|_{\mathscr{A}} \\
& \quad \leq\left\|\mathbf{w}_{1}-\mathbf{w}_{2}\right\|_{\mathscr{A}} K .
\end{aligned}
$$

Given any positive small number $\epsilon$, there exists a small number $\delta:=\varepsilon / K$, such that, for any $\mathbf{w}_{1}, \mathbf{w}_{2} \in \mathscr{C}^{p}(\mathscr{A})$, if $\left\|\mathbf{w}_{1}-\mathbf{w}_{2}\right\|_{\mathscr{A}} \leq \delta$, then

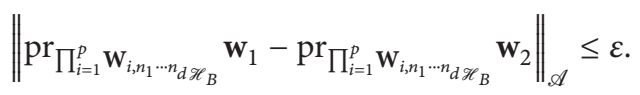

\section{Competing Interests}

The authors declare that they have no competing interests.

\section{Acknowledgments}

The authors wish to thank the Ministry of Research, Technology and Higher Education (RISTEK-DIKTI) for the financial 
support. They also thank Karlsruher Institut für Technologie (KIT) Institut für Stochastik for hospitality. Special thanks are addressed to Professor Andrei I. Volodin for his constructive comments for the improvement of the paper.

\section{References}

[1] A. Zellner, "An efficient method of estimating seemingly unrelated regressions and tests for aggregation bias," Journal of the American Statistical Association, vol. 57, no. 298, pp. 348-368, 1962.

[2] R. Christensen, Advanced Linear Modeling: Multivariate, Time Series, and Spatial Data; Nonparametric Regression and Response Surface Maximization, Springer, New York, NY, USA, 2001.

[3] D. W. Anderson, An Introduction to Multivariate Statistical Analysis, John Wiley \& Sons, New York, NY, USA, 3rd edition, 2003.

[4] R. A. Johnson and D. W. Wichern, Applied Multivariate Statistical Analysis, Prentice Hall, New York, NY, USA, 3rd edition, 2007.

[5] I. B. MacNeill, "Properties of partial sums of polynomial regression residuals with applications to test for change of regression at unknown times," The Annals of Statistics, vol. 6, no. 2, pp. 422-433, 1978.

[6] I. B. MacNeill, "Limit processes for sequences of partial sums of regression residuals," The Annals of Probability, vol. 6, no. 4, pp. 695-698, 1978.

[7] I. B. MacNeill and V. K. Jandhyala, "Change-point methods for spatial data," in Multivariate Environmental Statistics, G. P. Patil and C. R. Rao, Eds., pp. 298-306, Elevier Science, Berlin, Germany, 1993.

[8] L. Xie and I. B. MacNeill, "Spatial residual processes and boundary detection," South African Statistical Journal, vol. 40, no. 1, pp. 33-53, 2006.

[9] W. Bischoff and W. Somayasa, "The limit of the partial sums process of spatial least squares residuals," Journal of Multivariate Analysis, vol. 100, no. 10, pp. 2167-2177, 2009.

[10] W. Somayasa, Ruslan, E. Cahyono, and L. O. Ngkoimani, "Checking adequateness of spatial regressions using setindexed partial sums technique," Far East Journal of Mathematical Sciences, vol. 96, no. 8, pp. 933-966, 2015.

[11] W. Bischoff, "A functional central limit theorem for regression models," The Annals of Statistics, vol. 26, no. 4, pp. 1398-1410, 1998.

[12] W. Bischoff, "The structure of residual partial sums limit processes of linear regression models," Theory of Stochastic Processes, vol. 2, pp. 23-28, 2002.

[13] W. Stute, "Nonparametric model checks for regression," The Annals of Statistics, vol. 25, no. 2, pp. 613-641, 1997.

[14] W. Stute, W. González Manteiga, and M. Presedo Quindimil, "Bootstrap approximations in model checks for regression," Journal of the American Statistical Association, vol. 93, no. 441, pp. 141-149, 1998.

[15] E. J. Bedrick and C.-L. Tsai, "Model selection for multivariate regression in small samples," Biometrics, vol. 50, no. 1, pp. 226231, 1994.

[16] Y. Fujikoshi and K. Satoh, "Modified AIC and $C_{p}$ in multivariate linear regression,” Biometrika, vol. 84, no. 3, pp. 707-716, 1997.

[17] W. Bischoff and A. Gegg, "Partial sum process to check regression models with multiple correlated response: with an application for testing a change-point in profile data," Journal of Multivariate Analysis, vol. 102, no. 2, pp. 281-291, 2011.

[18] W. Somayasa and G. N. Adhi Wibawa, "Asymptotic modelcheck for multivariate spatial regression with correlated responses," Far East Journal of Mathematical Sciences, vol. 98, no. 5, pp. 613-639, 2015.

[19] P. Billingsley, Convergence of Probability Measures, John Wiley \& Sons, New York, NY, USA, 1968.

[20] M. Tahir, "Prediction of the amount of nickel deposit based on the results of drilling bores on several points (case study: south mining region of PT. Aneka Tambang Tbk., Pomalaa, Southeast Sulawesi)," Research Report, Halu Oleo University, Kendari, Indonesia, 2010.

[21] K. V. Mardia and C. R. Goodall, "Spatial-temporal analysis of multivariate environmental monitoring data," in Multivariate Environmental Statistics, G. P. Patil and C. R. Rao, Eds., pp. 347386, North-Holland, Amsterdam, The Netherlands, 1993.

[22] S. Arnold, "The asymptotic validity of invariant procedures for the repeated measures model and multivariate linear model," Journal of Multivariate Analysis, vol. 15, no. 3, pp. 325-335, 1984.

[23] R. Pyke, "A uniform central limit theorem for partial sum processes indexed by sets," in London Mathematical Society Lecture Note Series, vol. 79, pp. 219-240, 1983.

[24] R. F. Bass and R. Pyke, "Functional law of the iterated logarithm and uniform central limit theorem for partial-sum processes indexed by sets," The Annals of Probability, vol. 12, no. 1, pp. 1334, 1984.

[25] K. S. Alexander and R. Pyke, "A uniform central limit theorem for set-indexed partial-sum processes with finite variance," The Annals of Probability, vol. 14, no. 2, pp. 582-597, 1986.

[26] D. W. Stroock, A Concise Introduction to the Theory of Integration, Birkhäuser, Berlin, Germany, 3rd edition, 1999.

[27] J. N. Magnus and H. Neudecker, Matrix Differential Calculus with Applications in Statistics and Econometrics, John Wiley \& Sons, New York, NY, USA, 3rd edition, 2007.

[28] K. B. Athreya and S. N. Lahiri, Measure Theory and Probability Theory, Springer, New York, NY, USA, 2006.

[29] A. W. van der Vaart, Asymptotic Statistics, vol. 3, Cambridge University Press, London, UK, 1998.

[30] E. L. Lehmann and J. P. Romano, Testing Statitical Hypotheses, Springer, New York, NY, USA, 3rd edition, 2005.

[31] H. Pruscha, Vorlesungen über Mathematische Statistik, B.G. Teubner, Stuttgart, Germany, 2000.

[32] J. Shao and D. S. Tu, The Jackknife and Bootstrap, Springer, New York, NY, USA, 1995.

[33] J. Yeh, "Cameron-Martin translation theorems in the Wiener space of functions of two variables," Transactions of the American Mathematical Society, vol. 107, no. 3, pp. 409-420, 1963.

[34] J. Elstrodt, Maß- und Integrationstheorie, vol. 7 of Korregierte und Aktualisierte Auflage, Springer, Berlin, Germany, 2011. 


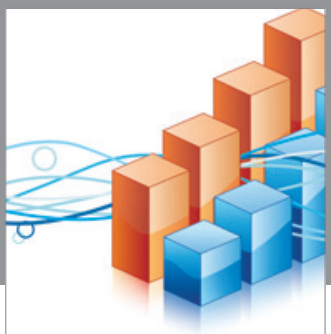

Advances in

Operations Research

vatem alat4

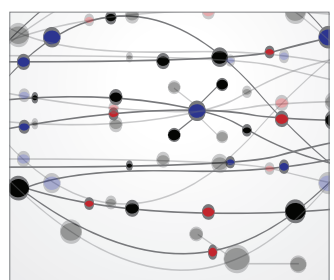

\section{The Scientific} World Journal
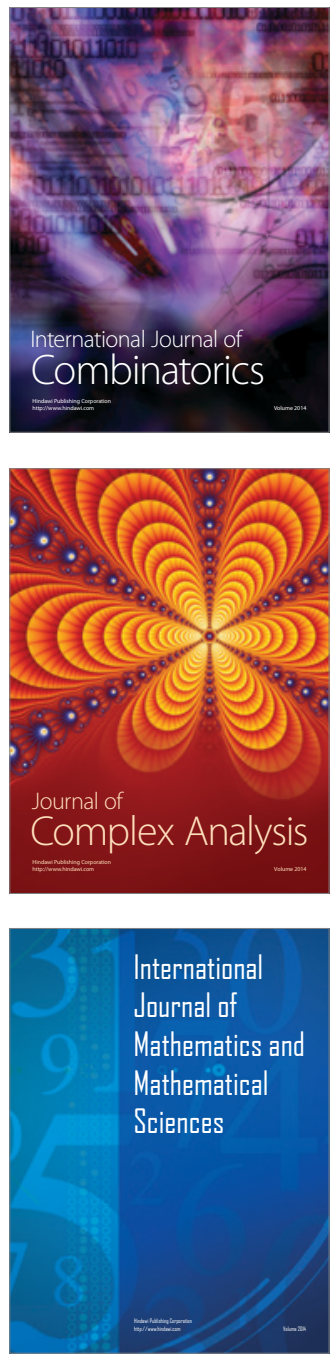
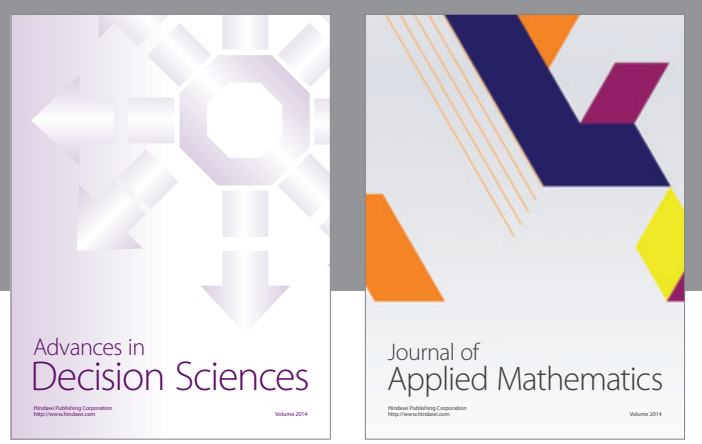

Algebra

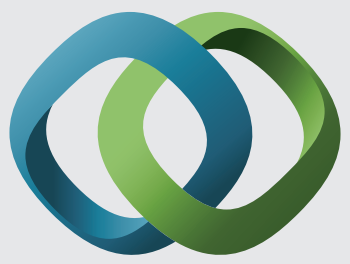

\section{Hindawi}

Submit your manuscripts at

http://www.hindawi.com
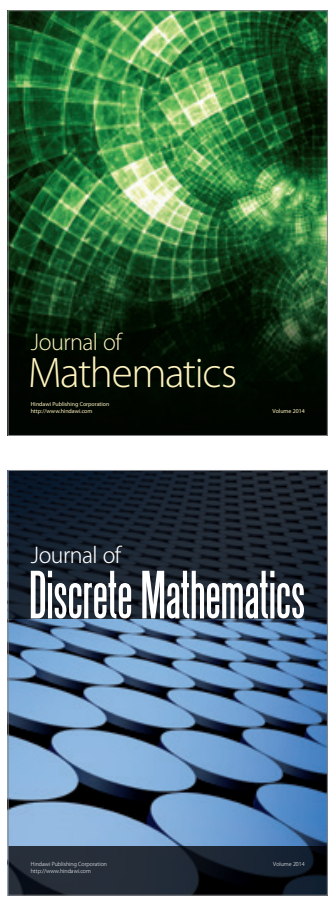

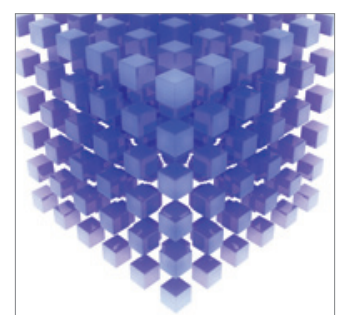

Mathematical Problems in Engineering
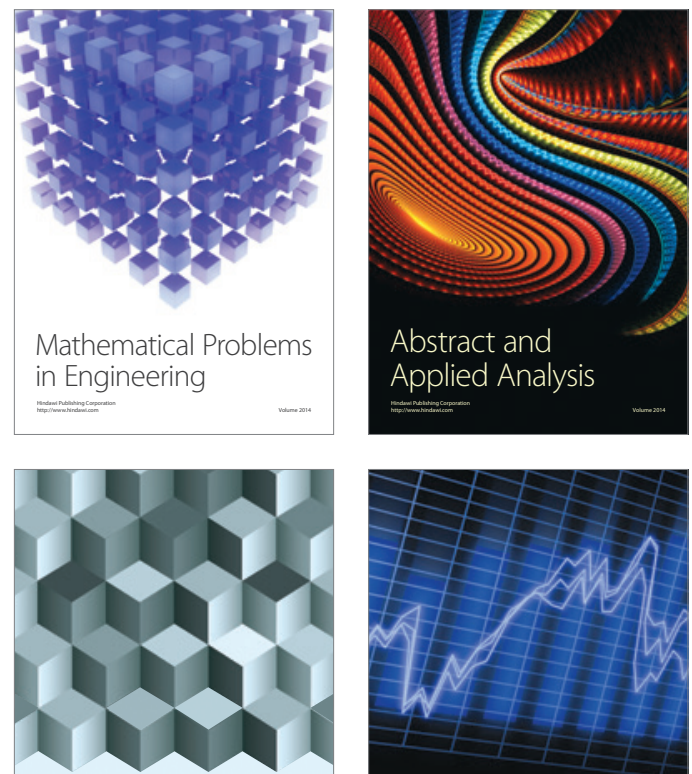

Journal of

Function Spaces

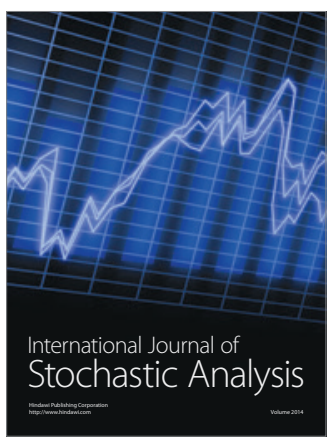

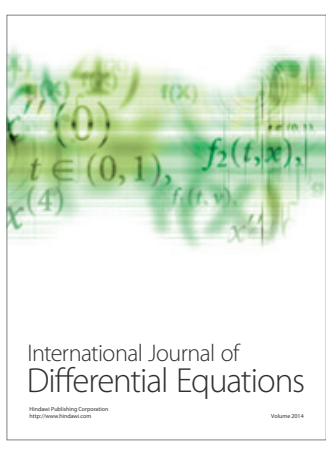
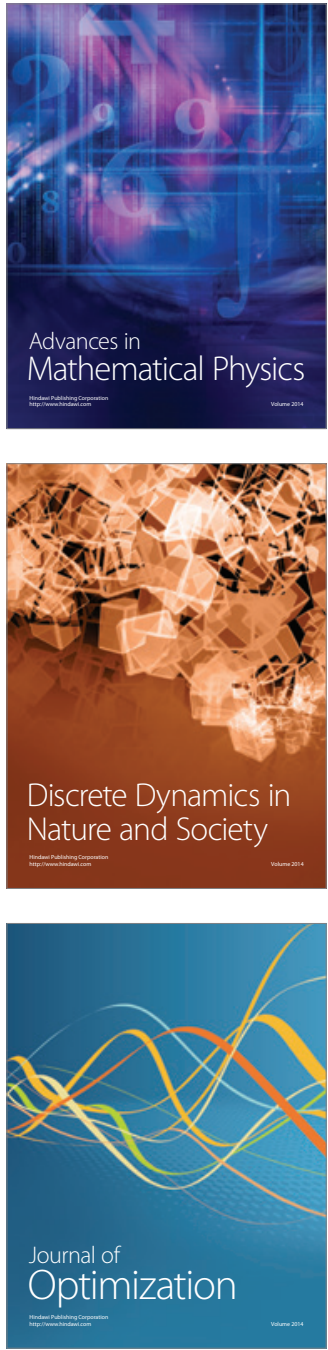